\title{
Fasciola gigantica, $F$. hepatica and Fasciola intermediate forms: geometric morphometrics and an artificial neural network to help morphological identification
}

\author{
Suchada Sumruayphol ${ }^{\text {Equal first author, } 1}$, Praphaiphat Siribat ${ }^{\text {Equal first author, } 2}{ }^{2}$, Jean-Pierre Dujardin ${ }^{3}$, Sébastien Dujardin ${ }^{3}$, \\ Chalit Komalamisra ${ }^{4}$, Urusa Thaenkham ${ }^{\text {Corresp. } 5}$ \\ ${ }^{1}$ Department of Medical Entomology, Faculty of Tropical Medicine, Mahidol University, Bangkok, Thailand \\ 2 Department of Biochemistry, Faculty of Science, Mahidol University, Bangkok, Thailand \\ 3 IRD, UMR, INTERTRYP IRD, CIRAD, University of Montpellier, Montpellier, France \\ 4 Mahidol-Bangkok School of Tropical Medicine, Faculty of Tropical Medicine, Mahidol University, Bangkok, Thailand \\ 5 Department of Helminthology, Faculty of Tropical Medicine, Mahidol University, Bangkok, Thailand \\ Corresponding Author: Urusa Thaenkham \\ Email address: urusa.tha@mahidol.ac.th
}

Background. Fasciola hepatica and F. gigantica cause fascioliasis in both humans and livestock. Some adult specimens of Fasciola sp. referred to as "intermediate forms" based on their genetic traits, are also frequently reported. Simple morphological criteria are unreliable for their specific identification. In previous studies, promising phenotypic identification scores were obtained using morphometrics based on linear measurements (distances, angles, curves) between anatomical features. Such an approach is commonly termed "traditional" morphometrics, as opposed to "modern" morphometrics, which is based on the coordinates of anatomical points. Methods. Here, we explored the possible improvements that modern methods of morphometrics, including landmark-based and outline-based approaches, could bring to solving the problem of the non-molecular identification of these parasites. F. gigantica and Fasciola intermediate forms suitable for morphometric characterization were selected from Thai strains following their molecular identification. Specimens of $F$. hepatica were obtained from the Liverpool School of Tropical Medicine (UK). Using these three taxa, we tested the taxonomic signal embedded in traditional linear measurements versus the coordinates of anatomical points (landmarkand outline-based approaches). Various statistical techniques of validated reclassification were used, based on either the shortest Mahalanobis distance, the maximum likelihood, or the artificial neural network method. Results. Our results revealed that both traditional and modern morphometric approaches can help in the morphological identification of Fasciola sp. We showed that the accuracy of the traditional approach could be improved by selecting a subset of characters among the most contributive ones. The influence of size on discrimination by shape was much more important in traditional than in modern Peerj reviewing PDF | (2019:08:40125:2:0:NEW 7 Jan 2020) 
analyses. In our study, the modern approach provided different results according to the type of data: satisfactory when using pseudolandmarks (outlines), less satisfactory when using landmarks. The different reclassification methods provided approximately similar scores, with a special mention to the neural network, which allowed improvements in accuracy by combining data from both morphometric approaches. Conclusion. We conclude that morphometrics, whether traditional or modern, represent a valuable tool to assist in Fasciola species recognition. The general level of accuracy is comparable among the various methods, but their demands on skills and time differ. Based on the outline method, our study could provide the first description of the shape differences between species, highlighting the more globular contours of the intermediate forms. 
1 Fasciola gigantica, $F$. hepatica and Fasciola intermediate forms: geometric

2 morphometrics and an artificial neural network to help morphological

\section{3 identification}

4

5 Suchada Sumruayphol ${ }^{1, *}$, Praphaipat Siribat ${ }^{2,5, *}$, Jean-Pierre Dujardin ${ }^{3}$, Sébastien Dujardin ${ }^{3}$,

6 Chalit Komalamisra ${ }^{4}$, Urusa Thaenkham ${ }^{5}$

$7{ }^{1}$ Department of Medical Entomology, Faculty of Tropical Medicine, Mahidol University,

8 Bangkok 10400, Thailand

92 Department of Biochemistry, Faculty of Science, Mahidol University, Bangkok 10400,

10 Thailand

$11{ }^{3}$ IRD, UMR INTERTRYP IRD, CIRAD, University of Montpellier, F-34398 Montpellier,

12 France.

$13{ }^{4}$ Mahidol-Bangkok School of Tropical Medicine, Faculty of Tropical Medicine, Mahidol

14 University, Bangkok, Thailand

$15{ }^{5}$ Department of Helminthology, Faculty of Tropical Medicine, Mahidol University, Bangkok

16 10400, Thailand

$17 *$ These authors contributed equally to this work

18 Corresponding Author:

19 Urusa Thaenkham ${ }^{5}$

20 420/6 Ratchawithi Road, Ratchathewi, Bangkok, Thailand

21 Email address: urusa.tha@mahidol.ac.th 


\section{ABSTRACT}

26 Background. Fasciola hepatica and F. gigantica cause fascioliasis in both humans and livestock.

27 Some adult specimens of Fasciola sp. referred to as "intermediate forms" based on their genetic 28 traits, are also frequently reported. Simple morphological criteria are unreliable for their specific identification. In previous studies, promising phenotypic identification scores were obtained using morphometrics based on linear measurements (distances, angles, curves) between anatomical features. Such an approach is commonly termed "traditional" morphometrics, as opposed to "modern" morphometrics, which is based on the coordinates of anatomical points.

Methods. Here, we explored the possible improvements that modern methods of morphometrics, including landmark-based and outline-based approaches, could bring to solving the problem of the non-molecular identification of these parasites. F. gigantica and Fasciola intermediate forms suitable for morphometric characterization were selected from Thai strains following their molecular identification. Specimens of $F$. hepatica were obtained from the Liverpool School of Tropical Medicine (UK). Using these three taxa, we tested the taxonomic signal embedded in traditional linear measurements versus the coordinates of anatomical points (landmark- and outline-based approaches). Various statistical techniques of validated reclassification were used, based on either the shortest Mahalanobis distance, the maximum likelihood, or the artificial neural 42 network method.

43 Results. Our results revealed that both traditional and modern morphometric approaches can help 44 in the morphological identification of Fasciola sp. We showed that the accuracy of the traditional approach could be improved by selecting a subset of characters among the most contributive ones. 
46 The influence of size on discrimination by shape was much more important in traditional than in 47 modern analyses. In our study, the modern approach provided different results according to the 48 type of data: satisfactory when using pseudolandmarks (outlines), less satisfactory when using 49 landmarks. The different reclassification methods provided approximately similar scores, with a 50 special mention to the neural network, which allowed improvements in accuracy by combining 51 data from both morphometric approaches.

52 Conclusion. We conclude that morphometrics, whether traditional or modern, represents a 53 valuable tool to assist in Fasciola species recognition. The general level of accuracy is comparable 54 among the various methods, but their demands on skills and time differ. Based on the outline 55 method, our study could provide the first description of the shape differences between species, 56 highlighting the more globular contours of the intermediate forms. 


\section{INTRODUCTION}

58 Fasciola hepatica Linnaeus, 1758 and F. gigantica Cobbold, 1855 are well-known as parasites

59 that cause fascioliasis (Mas-Coma, Bargues \& Valero, 2005). The parasites belong to the family

60 Fasciolidae (Railliet, 1895), subfamily Fasciolinae (Stiles \& Hassall, 1898). The adult worm

61 infects the liver of various species of mammals, particularly livestock that consume fresh plant

62 material contaminated with infective-stage metacercaria (Mas-Coma, Bargues \& Valero 2018).

63 Climatic changes seem to have exacerbated the problem by expanding the geographic distribution

64 of such parasites (Mas-Coma, Valero \& Barynes 2008; 2009a). Typically, F. hepatica is reported

65 worldwide, while $F$. gigantica occurs in a narrower range across Asia and Africa. The geographic

66 distribution of these two parasites can overlap, particularly in the equatorial zone. The hybrid

67 forms that can result between $F$. hepatica and F. gigantica are often reported as Fasciola 68 “intermediate forms" (Periago et al., 2008; Mas-Coma, Valero \& Barynes 2009a; 2009b). Like

69 other countries in the tropical and subtropical areas, Thailand has reported Fasciola infections in 70 both humans and livestock (Mas-Coma, Valero \& Barynes 2009a; Buranasin \& Harinasuta, 1970;

71 Srihakim \& Pholpark, 1991; Aroonroch et al., 2006; Kanoksil et al., 2006; Tippawangkosol et al., 72 2017; Wannasan et al., 2014).

73

74 Up until now, traditional morphometrics, which is based on linear measurements between 75 anatomical landmarks, has been used to help in the identification of Fasciola species (Valero, 76 Panova \& Mas-Coma, 2005). Many factors have been noted which can modify the accuracy of 77 phenotypical classification done in this way, including: 1) lymnaeid-host specificity, 2) bias arising 78 from different types of definitive hosts, the technical bias for worm fixing, 3) the computerized 79 image analysis system used, 4) the standardized methodology used for measurements, and 5) the 
80 effect of allometric growth (Valero, Panova \& Mas-Coma, 2005; Periago et al., 2006). Size and

81 some other variables or ratios that have been erroneously considered as a proxy for "shape" (body

82 roundness $(\mathrm{BR}=\mathrm{BP} 2 / 4 \pi \mathrm{BA} ; \mathrm{BP}=$ body perimeter; and $\mathrm{BA}=$ body area $)$ could discriminate

83 between adult $F$. hepatica, F. gigantica, and Fasciola intermediate forms collected from different

84 countries in Asia (Periago et al., 2006), as well as being used to examine intraspecific diversity

85 (Periago et al., 2007). A molecular identification technique was developed to discriminate

86 between fasciolid adult worms based on ribosomal internal transcribed spacers (ITS) 1 and 2 (Lin

87 et al., 2007; Lotfy et al., 2008). According to Amer et al. (2011), the identification of fasciolids

88 requires at least ITS2 and mitochondrial cox1 or nad1 sequences. Itagaki et al. (2011) suggested

89 that ITS1 sequences, which do not show variation in Europe or Oceania, are nonetheless useful

90 molecular markers for discriminating F. hepatica, F. gigantica, and their hybrids occurring in Asia

91 (Amer et al., 2011).

92

93 In Thailand, Fasciola intermediate forms were found in the livers of cattle in slaughterhouses and

94 identified as hybrids because of the heterosequences of nuclear ribosomal ITS1 and ITS2

95 (Chaichanasak et al., 2012; Siribat et al., 2018). For molecular discrimination between $F$.

96 gigantica, F. hepatica, and Fasciola intermediate forms, ITS1 has been noted as the most effective

97 DNA marker (Chaichanasak et al., 2012). Fasciola intermediate forms present no qualitative

98 morphological attributes that allow clear identification. Alongside molecular discrimination

99 techniques, traditional morphometrics has been suggested as a relatively simple and effective

100 approach for species identification (Periago et al., 2006; Valero, Marcos \& Mas-Coma, 1996;

101 Valero, Panova \& Mas-Coma, 2005; Ashrafi et al., 2006; Afshan et al., 2014). However, the 
102 dimensions of Fasciola intermediate forms may overlap with those of both $F$. gigantica and $F$.

103 hepatica (Periago et al., 2006; Srimuzipo et al., 2000).

104

105 Even when using many characters and multivariate statistical tools, the risk of using a 106 morphometrics method is that linear distance measurements mainly provide information relating 107 to size and provide limited information about shape. Previous studies inaccurately labeled as 108 "shape" some variables which are actually another estimation of size, such as body roundness (BR $\left.109=\mathrm{BP}^{2} / 4 \pi \mathrm{BA}\right)$, body perimeter (BP), and body area (BA) (Periago et al., 2006; Mas-Coma, Valero 110 \& Bargues 2009b; Valero et al., 2018). These characters are secondary constructions from linear 111 measurements and produce variables redundant with size (Burnaby, 1966).

113 A more convenient approach has recently been applied which enables variations in shape to be 114 estimated from linear measurements (Valero et al., 2018). However, there are various techniques 115 available that can be used for this. A comprehensive review was recently published distinguishing 116 two "schools" via which it is possible to approach the allometric concept: the Huxley-Jolicoeur 117 and the Gould-Mosimann schools (Klingenberg, 2016). The former, initially developed for 118 bivariate analyses, considers morphological form as a single unified feature (form as both size and 119 shape), emphasizing the covariation among traits as a consequence of variation in size. The latter, 120 initially developed for multivariate morphometric measurements, separates two estimates: an 121 estimate of global size and a multivariate estimate of shape (Mosimann, 1970; Darroch and 122 Mosimann, 1985). According to Klingenberg (2016), landmark-based geometric morphometrics 123 represents a straightforward extension to this second method. Its concept may be naturally 124 extended to the outline-based approach, which also separates a global estimator of size and a 
125 multivariate estimate of shape (Rohlf \& Archie, 1984; Lestrel, 1989). Instead of using distances

126 between anatomical landmarks, the modern methods use their coordinates in a common system of

127 axes. One of the clear improvements introduced by the modern approach is that it enables a visual

128 representation of shape (Rohlf \& Marcus, 1993). Visualization of changes in shape are frequently

129 presented using D'Arcy Thompson-like plots, or deformation grids (Bookstein, 1991).

130 Deformation grids help to visualize the shifts in aligned coordinates from one shape to another.

131 The grid is a visual aid and can even be amplified to improve the visibility of subtle changes. It

132 can be omitted when these changes are clearly visible, as is frequently the case between species,

133 without the need for any amplification artefacts.

135 The use of coordinates usually applies to animal species presenting a solid structure on their body.

136 However, this method has also been applied to soft structures. For example, when combined with

137 photogrammetric techniques, it can reveal variations in the shape of rodent sperm-cell heads and 138 the diversity of these shape variations among species (Varea Sánchez, Bastir \& Roldan, 2013).

140 In our study, we used recommended traditional morphometric techniques and, to preserve the 141 parallelism of shape extraction from the data, we applied the Darroch \& Mosimann technique 142 (1985) to the linear measurements, which produces "log-shape ratios" as estimates of shape.

143 We compared the results obtained by traditional techniques with those obtained from two methods

144 based on the coordinates of anatomical points: the landmark-based (Rohlf, 1990) and the outline145 based (Kuhl \& Giardina, 1982; Lestrel, 1997) approaches. These two approaches are conceptually 146 different. The landmark-based approach uses anatomical points called "landmarks". These points 147 are small biological structures, as small as a point at the requisite scale. They are considered to be 
148 homologous in the sense that they are relocatable points from one individual to another. The

149 outline-based approach also uses an ordered set of discrete point coordinates; these are

150 superficially similar to landmark data, but they are conceptually quite different. They are of a

151 different nature to true landmarks because comparability between them is not expected separately,

152 but from the structure they describe. The outline-based method is generally restricted to closed

153 contours (outlines) where landmarks are lacking. In this approach, the contour is described by

154 points that we call "pseudolandmarks" (Dujardin, 2017). There is no need for these

155 pseudolandmarks to be equally spaced, and there is no need to have the same number of them from

156 one individual to another (Rohlf, 1990a; Dujardin et al. 2014).

157

158 Pseudolandmarks should not be confused with "semilandmarks". Semilandmarks are landmarks

159 that lack one degree of freedom because they depend on other landmarks. They are generally used

160 to compare open curves between other landmarks, and, most importantly, they are processed as

161 true landmarks by Procrustes superposition, combined with a sliding procedure to minimize total

162 bending energy or to minimize the Procrustes distances to the consensus (Dujardin, 2019).

163

164 Outline data are processed in a very different way, either by elliptic Fourier analysis $(K u h l \&$

165 Giardina, 1982) or, less frequently, by eigenshape analysis (Lohmann, 1983). Briefly, the observed

166 contour is decomposed in terms of sine and cosine curves of successive frequencies called

167 harmonics, with each harmonic being described by four coefficients. These coefficients are

168 normalized by the semi-grand axis of the first harmonic ellipse. The latter may be used as an

169 estimate of global size, as well as the root-squared area of this same starting ellipse, the root-

170 squared area of the digitized contour, or its perimeter. We explored the capacity of these different 
171 approaches to discriminate between F. hepatica, F. gigantica, and Fasciola intermediate forms.

172

173 To ensure correct evaluation of the methods, the samples collected from Thailand were also

174 identified using molecular analysis. In addition to the Thailand samples, nine $F$. hepatica strains

175 from the UK were provided and used to give reference data: they were subjected to morphometric 176 analyses only.

177

178 As far as we know, morphometrics applied to the Fasciola identification always relied on 179 traditional morphometric techniques. In this study, we tried to answer the following question: 180 could modern morphometric techniques bring any improvement over traditional ones in terms of 181 accuracy and/or simplicity?

183 Materials \& methods

184 Sample collection

185 Ninety adult worms of Fasciola spp. were collected from the livers of cattle slaughtered in local 186 slaughterhouses in Lamphun, Mae Hong Son, Tak, and Pratum Thani Provinces, Thailand. The 187 cattle in the Pratum Thani slaughterhouse were from Kanchanaburi Province, Thailand. All cattle 188 came from local farms in Thailand. Infected livers were stored on ice and transferred to the 189 laboratory at the Department of Helminthology, Faculty of Tropical Medicine, Mahidol 190 University, within 12 hours and within 1 hour for the samples from Pratum Thani province. Adult 191 Fasciola worms were gently separated from the liver tissue. Each individual worm was fixed 192 between two transparent glass slides containing two pieces of paper $(0.4 \mathrm{~mm})$ and the two slides 193 were gently held in place using a rubber band. The specimens were placed in normal saline for 1 
194 hour. The lateral part of the body of each adult worm was then cut away and stored individually in 195 absolute alcohol at $-20^{\circ} \mathrm{C}$ until being used for the molecular study. The remaining section of each 196 worm's body was washed in distilled water for 5 min to relax the structure before being placed 197 individually between glass slides. The fixed adult worms were tentatively identified under a 198 stereomicroscope using the taxonomic criteria of primary morphological characters, including 199 cephalic cone, body length, and body width (Periago et al., 2008; Valero, Marcos \& Mas-Coma, 200 1996). The specimens were fixed in $70 \%$ ethanol and stored at $4{ }^{\circ} \mathrm{C}$ for staining.

201

202 In addition to our field specimens, nine $F$. hepatica specimens were obtained from the Liverpool 203 School of Tropical Medicine (UK). These specimens were used for comparison and subjected to 204 both traditional and geometric morphometric examination. All specimens of $F$. hepatica, which 205 had sheep as their definitive host, were received as permanently mounted slides and deposited in 206 our laboratory in 1993. They were stained with Semichon's acid-carmine and mounted with 207 Canada balsam.

208

209 To make sure that our relatively small samples did not generate outlier values, we 210 compared graphically the observed means obtained using our material to values already published 211 for the same hosts in different countries (Valero, Panova \& Mas-Coma, 2005; Halakou et al., 212 2017; Shafiei et al., 2014; Iyiola et al., 2018).

213

214 Molecular identification by PCR-RFLP

215 A small piece of adult Fasciola worm was cut from the lateral part of the body. Genomic DNA 216 was extracted from each individual using a Tissue Genomic DNA Mini Kit (Geneaid, Taipei, 
217 Taiwan), according to the manufacturer's protocol. The PCR amplicons of the ribosomal ITS1 and 218 ITS2 regions were amplified using the primers ITS1-F and ITS1-R for ITS1 and 3S and BD2 for 219 ITS2 (Siribat et al., 2018; Ichikawa \& Itagaki, 2010; Huang et al., 2004). We conducted PCR 220 amplification of the ITS1 and ITS2 regions three times for each sample. Each PCR reaction was 221 performed in $50 \mathrm{~mL}$ final volume composed of $1 \times$ TopTaq Master Mix Kit (Qiagen, Hilden, 222 Germany) (1U TopTaq polymerase, $1.5 \mathrm{mM} \mathrm{MgCl}_{2}$, and TopTaq polymerase buffer), 20 pmol of 223 each primer, and $10 \mathrm{ng} / \mu \mathrm{L}$ genomic DNA template. The PCR cycles consisted of initial 224 denaturation at $95^{\circ} \mathrm{C}$ for $3 \mathrm{~min}$ followed by 29 cycles of denaturation at $95{ }^{\circ} \mathrm{C}$ for $45 \mathrm{~s}$, annealing 225 at $55^{\circ} \mathrm{C}$ for $30 \mathrm{~s}$, and extension at $72{ }^{\circ} \mathrm{C}$ for $60 \mathrm{~s}$. The final extension of each PCR reaction was 226 conducted at $72{ }^{\circ} \mathrm{C}$ for $8 \mathrm{~min}$.

227

228

After purifying the PCR products using the ethanol precipitation method, $500 \mathrm{ng}$ of purified PCR 229 products from each sample was digested with restriction enzymes RsaI and NlaIII for ITS1 and 230 ITS2, respectively (New England BioLab Inc., Massachusetts, USA) at $37^{\circ} \mathrm{C}$ for $150 \mathrm{~min}$. The enzymatic digestion was inactivated by heating at $80{ }^{\circ} \mathrm{C}$ for $20 \mathrm{~min}$. The final digested PCR amplicons were run on a $2 \%$ agarose gel at $50 \mathrm{~V}$ for $180 \mathrm{~min}$. The band patterns were visualized using a UV transilluminator. The PCR-RFLP figure was photographed with Gel Documentation 234 (G-Box (HR), Syngene, UK).

\section{Parasite staining}

237 The unfolded worms were stained overnight with Semichon's acid-carmine, then de-stained with $1 \%$ hydrochloric acid for $5 \mathrm{~min}$. The stained specimens were dehydrated in an ethanol dilution series of 70\%, 80\%, 90\% (twice), 95\% (twice), and finally in absolute ethanol (twice). Each 
240 dehydration step took $30 \mathrm{~min}$. The stained specimens were performed using 1:1 xylene and

241 absolute ethanol for $30 \mathrm{~min}$ before being transferred to pure xylene for $30 \mathrm{~min}$; by doing it twice.

242 Finally, the specimens were mounted with Canada balsam (Waikagul \& Thaenkham, 2014).

243

244 After staining, all specimens were photographed using a Nikon DS-Ri1 SIGHT digital camera 245 connected to a Nikon AZ 100 M stereomicroscope (Nikon Corp., Tokyo, Japan), and the scale was 246 indicated on each photograph. An initial classification was performed based on a visual 247 examination of the specimen's external morphology. The results of these identifications were 248 compared with the outcomes of the PCR-RFLP molecular identification using ITS1 and ITS2 as 249 the molecular markers (Table 1). After that, ImageJ software (freely available at 250 https://imagej.nih.gov/ij/) was used to measure the 20 morphological characters used in traditional 251 morphometric analyses (Table 2).

252

253 Data processing

254 Among the 74 and 16 genetically identified $F$. gigantica and Fasciola intermediates, respectively, 255 we selected the less damaged specimens that constituted the best samples for the morphometric 256 studies: $17 \mathrm{~F}$. gigantica and 10 Fasciola intermediate form specimens (from this study). In 257 addition, nine F. hepatica (from Liverpool School of Tropical Medicine, UK) were included in the 258 material.

259

260 Four types of morphometric data were separately analyzed: (i) log-transformed linear 261 measurements (LN), (ii) log-shape ratios (LSR), (iii) outlines data (OD), and (iv) landmarks data 262 (LD). For the artificial neural network-based classification (ANN, see below), we also tested the 
263

264

265

266

267

268

269

270

271

272

\section{Traditional morphometrics analyses}

274 Twenty characters were measured using a computerized image analysis system, as described by 275 Valero and colleagues (Valero, Marcos \& Mas-Coma, 1996; Valero, Panova \& Mas-Coma, 2005). 27 27 278

279 280 281 282 283 284 285

combination of LN with OD.

\section{Software}

ImageJ image analysis software was used to measure linear dimensions. Landmarks and pseudolandmarks (outlines) were digitized using the CLIC package (https://www.xyom-clic.eu). Subsequent analyses and graphical outputs were performed using both the CLIC package and the recently available online XYOM software (https://xyom.io) (Dujardin \& Dujardin, 2019). The latter allowed some specific analyses to be performed, including the maximum likelihood $(M x L)$ and the ANN methods of classification (see below).

Valero and colleagues (Valero, Marcos \& Mas-Coma,

We compared graphically our data with previously published ones, showing the departures of observed means (between the published means and ours) from the total means (Fig. S1).

Univariate morphometric comparisons used non-parametric techniques based on 1000 random permutations of the data between groups. The linear measurements (LN) were log-transformed for the multivariate analyses and validated classification. In addition, the eigenvectors of PC1 and PC2 were used to extract the variables that made the largest contribution $(>25 \%)$, resulting in a total of 12 variables instead of 20. These were BL, BW, BP, CL, Vsmin, A-VS, OS-VS, VS-Vit, Vit-P, VS-P, TW, and TP (see Table 2). Log-linear measurements were then subjected to the Darroch \& Mosimann (1985) procedure to extract an estimate of global size (log-size) and an estimate of 
286 shape variables (log-shape ratios, LSR). The influence of size on interspecific discrimination by

287 LSR was estimated by regressing on log-size the discriminant factors.

288

289 Outline data (OD)

290 The contour of each specimen was manually digitized (see Fig. 1A), and the resulting coordinates 291 were subjected to an elliptic Fourier analysis. The "outline data" (OD) used as the input for 292 validated classification were the normalized elliptic Fourier coefficients (NEF). Their numbers 293 were restricted to an average set giving a power of at least $99 \%(n=30)$. Their first eight principal 294 components were used directly as input for the validated classification tests. An estimate of the 295 allometric residue contained in shape variables was performed by regressing the principal 296 components of the NEF on the square root of the area delimited by the contours. The non297 parametric estimation of the statistical significance of this multivariate regression followed the 298 procedure of Good (2000). On the other hand, the influence of the global size on interspecific 299 discrimination itself was estimated after regressing the first discriminant factor on size. For the 300 artificial neural network technique (ANN, see below), the OD were also combined with different 301 numbers $(4,6,8,10$, and 12) of LN.

302

303 Landmarks data (LD)

304 The following anatomical landmarks (LM) were digitized: LM1 = left-side of the cephalic cone, 305 LM2 = left-side of the oral sucker, LM3 = right-side of the oral sucker, LM4 = right-side of the 306 cephalic cone, and LM5 = end of the testis (see Fig. 1B). The five landmarks were subjected to a 307 generalized Procrustes analysis, and the resulting aligned individuals were orthogonally projected 308 onto the Euclidean space tangent to the consensus of forms (Rohlf \& Slice, 1990). The principal 
309 components (PC) of these data (called "Procrustes residuals", "tangent space variables", or 310 "orthogonal projections") were referred to here as "landmarks data" (LD) and used as the input for

311 validated classification. The influence of size on interspecific discrimination by LD was estimated

312 by regressing the discriminant factors on the centroid size.

313

314 Measurement error due to imaging and digitizing

315 The precision of our digitization was evaluated for both landmarks and outlines. For size we used 316 the method recommended by Arnqvist \& Mårtensson (1998), and for shape we used the Procrustes 317 analysis of variance (Procrustes ANOVA) (Klingenberg and McIntyre, 1998).

\section{Validated classification}

To assess how our results could be generalized to an independent data set, each individual was iteratively removed and its assignation computed following the analysis of the remaining data (the "leave-one-out" method, also known as "validated reclassification") (see Supplementary data 1). The analyses of the remaining data used three different statistical tests: a distance-based (Dist) method, a maximum likelihood $(M x L)$ method, and an ANN method.

325

326

In the first method (Dist), the Mahalanobis distance was computed between the testing set (one 327 individual) and the average of each training group (the remaining data), and the tested individual was assigned to the closest group. In the second method $(M x L)$, the testing set (one individual) was assigned to the group it was thought most likely to belong to assuming a normal distribution and independence between characters (Polly \& Head, 2004; Dujardin et al., 2017). For the Dist and $M x L$ methods we tried to avoid using a number of input variables that would exceed the number 
332 of individuals in the smallest sample. There are various methods to deal with multidimensionality

333 and we selected the simplest one: the set of first PCs lower than the number of individuals in the

334 smallest sample. Note that for the analyses related to the use of five anatomical landmarks, the

335 total number of final shape variables was actually six (two-times the number of LM minus 4 in the 336 case of two-dimensional analyses).

337

338 The ANN method made use of a simple multilayer perceptron with a back propagation algorithm 339 (Rumelhart, Hinton \& Wiliams, 1988), a variant of the original perceptron model (Rosenblatt, 340 1958) that is not, however, restricted to linear models. The method has been recently applied to 341 morphometric data, including both OD (Mancuso, 1999) and LD (Dobigny, Baylac \& Denys, 342 2002; Baylac, Villemant \& Simbolotti, 2003; Van Bocxlaer \& Schultheiß, 2010; Lorenz, Ferraudo $343 \&$ Suesdek, 2015; Soda, Slice \& Naylor, 2017). We used the multilayer perceptron program written

344 in JavaScript, which is available at https://www.npmjs.com/package/mlp.

346 Following a process of trial-and-error, a single (hidden) layer composed of three neurons provided 347 the best results, irrespective of the type of input data. We used as the input the total number of 348 variables instead of a subset of their PCs. Inputs comprised the morphometric variables (LN, LSR, 349 OD, or LD, see above), while the final outputs were the probabilities of inclusion in each group 350 (in a Bayesian sense). Forms were then classified to the species for which their probability of 351 inclusion was the greatest (Soda et al., 2017). The "weights", i.e., the coefficients allowing the

352 outputs to be computed, were applied to the individuals being tested to compute categories and 353 suggest a classification. Thus, each individual was tentatively assigned to its putative species after 354 computing the weights from the remaining data. For a single individual to identify, the remaining 
355 data were subjected to a large number of iterations to reduce the classification error to a minimum

356 (less than 25\%). All specimens were separately classified 30 times, then an average classification

357 and its standard error were computed (see Supplementary data 1).

358

359 To make the most of the less stringent statistical assumptions of the ANN method with regard to

360 the statistical distribution of the input data (Ripley, 1996), we explored its capacity to deal with 361 different metrics combined into a single matrix. Thus, we built a matrix with four LN and made 362 another (normalized) matrix combining them with the OD. We performed a validated 363 reclassification on each matrix and then compared the scores with the correct identification. We 364 repeated this comparison successively using $6,8,10$, and finally $12 \mathrm{LN}$, combined with the same 365 set of OD.

366

367 RESULTS

368 External morphological classification

369 The rough identification of our 90 field specimens did not detect the presence of Fasciola 370 intermediate forms despite them being present, as determined by the molecular analyses (see next 371 paragraph). Moreover, the rough identification wrongly assigned $30 \%$ of the specimens as $F$. 372 hepatica species, despite the complete absence of this species, as revealed by the molecular data 373 (Table 1).

374

375 Molecular identification

376 The PCR amplicons of the ITS1 and ITS2 regions (680 and $550 \mathrm{bp}$ ) from 90 adult worms of

377 Fasciola spp. were digested by RsaI and NlaIII, respectively, and provided species-specific band 
378 patterns that enable the three Fasciola species to be distinguished (Fig. S2, S3). The results showed 379 that 74 worms were classified as $F$. gigantica and 16 worms were Fasciola intermediate forms. $F$. 380 hepatica was not present in the samples collected in the field in Thailand (Table 1).

381

382

383 384 385 386 387 388 389 390 391

\section{Traditional morphometrics (univariate analyses)}

Six out of 20 characters showed significant differences between the three species: CL, CW, VSmax, VSmin, A-VS, and OS-VS (Table 2). Between Fasciola intermediate forms and the other two species, BL, BP, OSmax, VS-Vit, VS-P, PhL, PhW, and TL were statistically significantly different. The remaining variables appeared to be similar among the three species (Table 2).

\section{Traditional morphometrics (multivariate analyses)}

Based on the complete set of 20 linear measurements, the validated classification produced excellent scores of correct species attribution, ranging from $89 \%$ to $92 \%$ according to the reclassification method (Table 3, column "20LN"). In order to tentatively reduce the number of characters, we selected a subset of contributing variables. We did not select them according to univariate statistical differences between groups but according to their contribution to the first and second principal components. This selection allowed us to restrict the set of LN from 20 to 12 variables (BL, BW, BP, CL, Vsmin, A-VS, OS-VS, VS-Vit, Vit-P, VS-P, TL, and TW). The exclusion of poorly contributing variables was beneficial and substantially improved the validated reclassification score from $88 \%$ to $97 \%$ (Table 3, column "12LN"). The shape variables derived from LN (either LSR from 20LN or LSR from 12LN) produced very similar scores as logtransformed raw variables (Table 3). 


\section{Allometry}

402 The influence of size on LSR-based discrimination was high: $61 \%$ on the first discriminant factor 403 (DF1) and 63\% on the second (DF2).

404

405

\section{Repeatability}

406 The precision of the global size estimation was almost perfect (99\%) when using both the landmark 407 and outline techniques. For shape, the precision was slightly lower when using the landmarks 408 technique (93\%) compared with the outlines technique (98\%).

409

\section{Global size}

411 The global size of the parasites, either the body perimeter as computed from OD, the square-root 412 area within the contour (Fig. 2A; Table 4a) or the centroid size as computed from LD (Fig. 2B, 413 Table 4b) differed significantly between the intermediate forms and the other two species. These 414 other two species, $F$. hepatica and $F$. gigantica, did not show statistically significant differences 415 in global sizes (Table 5). Fasciola intermediate forms had the smallest global size, both in terms 416 of centroid size and the perimeter or square-root area of the contour.

\section{Outline-based analyses}

\section{Shape variation among Fasciola spp. according to the outline-based analysis}

420 Direct visualization, without amplification, of contour differences suggested Fasciola intermediate 421 forms possess a more globular contour (Fig. 3A). The factor maps of the discriminant analyses 422 (Fig. 3B) showed non-overlapping distributions. Accordingly, satisfactory validated classification 423 scores were obtained, ranging from $89 \%$ to $94 \%$ (Table 3, column "OD"). 


\section{Allometry}

425 The contribution of global size variation to the first shape-derived discriminant factor derived from 426 OD was $23 \%$, while it was $6 \%$ for the second one. According to the non-parametric method used 427 to evaluate the multivariate regression significance between size and shape, the allometric residue 428 contained in OD shape variables was significant $(p<0.001)$.

\section{Landmark-based analyses}

431 The mean specimens aligned according to the five anatomical landmarks used here showed some 432 separation between the three forms, with a seemingly more critical difference for Fasciola 433 intermediate forms (Fig. 4A), as for the OD (Fig. 3). The factor map of the discriminant analysis 434 showed that Fasciola intermediate forms could diverge from $F$. gigantica and F. hepatica (Fig. 435 4B). However, some overlapping was observed, and the validated classification scores based on 436 landmark data were not satisfactory, ranging from $58 \%$ to a maximum of $69 \%$ (Table 3 , column 437 “LD”).

\section{Statistical significance of shape variation}

440 The pairwise Mahalanobis distances based on shape, for both landmark- and outline-based shapes, 441 were highly significant $(p<0.001)$ between Fasciola intermediate forms and F. gigantica or $F$. 442 hepatica. Between $F$. gigantica and $F$. hepatica, the distance was smaller, albeit almost significant $443(p \sim 0.06)$ (Table 6). 
446 The first and second discriminant factors derived from the Procrustes residuals were still under the

447 influence of size ( $42 \%$ and $4 \%$, respectively) after regression on centroid size, which was more 448 than for the outline-based analysis (23\% and $6 \%$, respectively) but much less than for the 449 traditional method (61\% and $63 \%$, respectively).

450

\section{Combination of log-transformed linear measurements and OD}

452 The ANN performance was significantly improved when traditional linear measurements (LN), 453 even a small number of them, were combined with outline shape descriptors (OD). This 454 performance could be appreciated by comparing the ANN accuracy when applied to these data 455 either separately or combined (Table 7). For instance, with ten traditional measurements as the 456 input, the ANN performance achieved $88 \%$ of correct identification, with OD only it reached $89 \%$

457 (Table 3), whereas by combining both types of data the total score jumped to 94\% (Table 7).

458

\section{DISCUSSION}

460 The specimens in this study were processed using the same treatment method. If some deformation 461 occurred as a result of this treatment, it is reasonable to expect that this would be the same for each 462 species. The average values of linear measurements of 12 selected morphological characters of 463 each Fasciola sp. were graphically compared with previously published values from the same 464 parasites and the same hosts (see Fig. S1). This comparison showed that our samples were in the 465 range of average values and suggested that the small number of specimens did not result in any 466 sample bias. 
468 To reduce the effects of growth on the phenotype, only fully mature stages were selected from 469 each molecularly defined group. However, in addition to possible growth heterogeneity, other 470 divergent influences could have affected phenotypic variations in our total sample, such as 471 different geographic origins, microhabitats, host, and seasonality. We considered that, within each 472 species or group, these environmental effects would probably have had much more of an impact 473 on size than on shape. We used the geometric morphometrics approach to tentatively set apart 474 these size effects from the morphometric data, extracting the remaining shape variables and using 475 them exclusively to describe the groups distinguished by molecular data. The allometric residue 476 that possibly remained in the shape variables was not removed because our comparisons concerned 477 species rather than intraspecific groups. Species may differ by their allometric growth, which 478 becomes part of their evolutionary divergence (Klingenberg, 2016).

479

480 Our tentative removal of the size effect from the linear measurements (either $20 \mathrm{LN}$ or $12 \mathrm{LN}$ ) did 481 not change the global classification scores as obtained by raw measurements, probably because it 482 could not perform a consistent removal of size, as indicated by our allometric analysis. Size 483 remained dominant in the LSR $(\sim 61 \%)$ relative to the PR $(\sim 41 \%)$ and OD $(\sim 24 \%)$. Another 484 inconvenience of LSR is that they do not allow a visual representation of shape changes between 485 taxa (Rohlf \& Marcus, 1993). Methods based on coordinates data allow such visual representation 486 (see Fig. 2D).

488 Our samples represented limited geographic isolates and, because of the low sample sizes, could 489 suffer from low representativity. Moreover, the number of variables could be greater than the 490 number of individuals in our smallest sample (nine $F$. hepatica). We followed the "rule" for a 
491 multivariate analysis, especially discriminant analysis, which is to use a number of variables that

492 does not exceed the number of individuals in the smallest sample. There are various methods

493 available to deal with multidimensionality. This species number of PC scores was selected so as

494 to use a number of variables that does not exceed the number of individuals in the smallest sample.

495 This procedure allowed multidimensionality to be reduced where necessary; this did not make our

496 samples more representative, but at least the information they provided was not over-interpreted.

497

498 Since linear measurements can provide information about both size and shape, and because of the

499 relatively small size of Fasciola intermediate forms (Fig. 2), it was expected that this sample could

500 be identified using traditional techniques. Nevertheless, linear measurements did not just reflect

501 global size variation since they could also allow an excellent score of recognition for the pair $F$.

502 hepatica and F. gigantica, which are parasites of similar global size (Fig. 2, Table 4). The

503 extraction of shape (LSR) did not produce different results, confirming the relatively poor

504 efficiency of shape removal from our linear measurements. With or without shape extraction,

505 traditional morphometrics appeared to be a powerful means of species determination. It does,

506 however, require an excellent knowledge of the parasite's internal anatomy.

507

508 The previously recommended number of linear measurements necessary to distinguish $F$. hepatica

509 from F. gigantica and Fasciola intermediate forms was as high as 20 (Valero, Panova \& Mas-

510 Coma, 2005; Ashrafi et al., 2006). By considering the contribution of each variable to their first

511 two PC, we were able to reduce this number from 20 to 12, with similar or better results (see Table

512 3). This improvement could be explained by the elimination of redundancy among measurements,

513 and a possible lack of precision for some of them. Either way, our examination of repeatability 
514 produced satisfactory results for both shape (93\%) and size (99\%), although these results were

515 lower than the results of the outline-based technique ( $98 \%$ and $99 \%$, respectively).

516

517 The outline-based morphometrics analysis was based on manual contour digitization. Although

518 this type of manual data collection is relatively fast, an automated capture technique might be

519 preferable. Automated curve tracing is already included in many types of imaging software, and it

520 may be more appropriate for generalizability (Yang et al., 2015), but its useful application is

521 dependent on the complexity of the contours (Sheets at al., 2006; Firmat et al., 2010). We would

522 recommend that a study be performed that is specifically aimed at comparing automatic and

523 manual capture techniques. The fact remains, however, that whether manual or automatic

524 techniques are used, the outline digitization process is a relatively easy task and does not require

525 highly specialized skills from the user.

526

527 The landmark-based approach required a little more knowledge about the internal anatomy of the

528 parasites and did not provide satisfactory results. The lower reclassification scores of the landmark

529 data relative to the OD should not be regarded as a rule. In our case, very few reliable LM could

530 be suggested, reducing the amplitude of shape capture, which could explain the unsatisfactory

531 scores obtained. It is possible that better results might be obtained with, for instance, a different

532 landmark configuration, or a different number of landmarks.

533

534 In our validated method of reclassification each individual was sequentially removed from the total

535 sample and its identification performed using the model computed without it, a method also called

536 a "leave-one-out" or "jack-knife" procedure (Manly, 1986). Thus, individual identification was 
537 repeated as many times as there were individuals in the total sample (see Supplementary data 1).

538 Our "accuracy" for each method (Tables 3 and 7) was the percentage of individuals that were

539 correctly identified at the end of each such procedure. For the ANN classification, each

540 classification session was repeated 30 times, and the accuracies were presented as the average and

541 standard deviation of the 30 sessions (Tables 3 and 7).

542

543 The validated classification applied to different kinds of data used different methods (Table 3).

544 The one based on the Mahalanobis distance (Dist) generally produced higher scores with more

545 correct identifications than the other classification techniques, which conforms with previous

546 studies and highlights the frequent relative superiority of this technique (Dujardin et al., 2017;

547 MacLoad et al., 2007; Sonnenschein et al., 2015). However, reliable classification and assessment

548 of group separation using this analysis is based on several assumptions, some of which frequently

549 do not coincide with morphometric data (Mitteroecker \& Bookstein, 2011). For this reason, we

550 added additional classification techniques, such as the simple maximum likelihood $(M x L)$

551 approach (Polly \& Head, 2004; Dujardin et al., 2017) and the ANN technique.

552

553 Since the $M x L$ method assumes normally distributed data and independence among the characters

554 used, our best results were obtained when applied to (a subset of) the PC of the log-transformed 555 input variables, rather than when applied to the raw variables (detailed comparisons not shown).

556 In agreement with previous comparisons (Dujardin et al., 2017), the MxL technique provided a 557 slightly lower accuracy than the Dist method.

558 
559 In our study, the ANN produced slightly lower scores than the Dist or the $M x L$ approaches. Our 560 idea was to compare the taxonomic signal embedded in different types of data rather than to deeply

561 explore the power of each classification method. So, for each classification method, we tried to

562 subject each type of data to the same process. The neural network was thus configured in the same 563 way, irrespective of which data were used as the input (see Supplementary data 2). Certainly, some

564 improvements could be obtained by refining the configuration according to the kind of data being 565 used. Moreover, a possible explanation for the relative weakness of the ANN method used here is the low sample sizes of the training data. The ANN method may require many data to achieve an

567 accurate level of performance (Soda, Slice \& Naylor, 2017). However, the general scores were 568 satisfactory, and we showed that this technique could greatly benefit from using combined data as

569 the input, for example using a few traditional measurements together with outline shape data 570 (Table 7).

571

572 CONCLUSION

573 Both traditional and modern morphometric approaches can be helpful in the morphological

574 identification of Fasciola sp. We showed that by reducing the number of linear measurements 575 from the 20 commonly recommended ones to a selected subset of 12 measurements, the accuracy 576 of identification could be improved.

577

578 In our study, the outline-based approach produced satisfactory classification scores, more so than

579 the landmark-based approach. Moreover, we showed for the first time the apparent differences in 580 shape between "intermediate forms" of Fasciola sp. and F. gigantica and F. hepatica. The relative 581 simplicity of data collection (even manual collection) makes the outline-based approach an 582 attractive alternative. 
583 By comparing various data collection and classification techniques, our study suggests promising 584 new strategies to follow in helping with the identification of species in the genus Fasciola based 585 on their morphology.

586

587 ACKNOWLEDGEMENTS

588 The authors would like to thank the Center Equipment Unit, Faculty of Tropical Medicine, 589 Mahidol University for supporting the excellent imaging system. We would like to thank Mr. Ulf 590 Biallas, Software Engineer, Axel Springer Ideas Engineering GmbH for his kind assistance.

591

592 ADDITIONAL INFORMATION AND DECLARATION

593 Funding

594 The work was financially supported for sample collection from the Department of Helminthology, 595 Faculty of Tropical Medicine, Mahidol University.

596 Competing Interest

597 The authors declare that they have no competing interests.

598 Author Contributions

599 - Suchada Sumruayphol analyzed the data using GM analyses and prepared the manuscript.

600 - Praphaipat Siribat photographed Fasciola spp., performed molecular works and prepared 601 the manuscript.

602

- Jean-Pierre Dujardin and Sebastien Dujardin analyzed the data using TM, GM and ANN

603 analyses and reviewed the final version of the manuscript.

604

- Chalit Komalamisra and Urusa Thaenkham supervised this study and reviewed the final

605 version of the manuscript.

606 All authors read and approved the final manuscript. 
607

608

609

610

61

612

613

614

615

616

617

618

619

620

621

622

623

624

625

626

627

628

\section{Data Availability}

The following information was supplied regarding data availability:

The raw data are provided as a Supplemental File

\section{Supplemental Information}

Supplemental information for this article can be found online at $\operatorname{xxxxxx}$

\section{REFERENCES}

Afshan K, Valero M, Qayyum M, Peixoto R, Magraner A, Mas-Coma S. 2014. Phenotypes of intermediate forms of Fasciola hepatica and F. gigantica in buffaloes from Central Punjab, Pakistan. Journal of Helminthology 88(4):417-426. DOI 10.1007/s11692-011-9109-8.

Amer S, Dar Y, Ichikawa M, Fukuda Y, Tada, C, Itagaki T, Nakai Y. 2011. Identification of Fasciola species isolated from Egypt based on sequence analysis of genomic (ITS1 and ITS2) and mitochondrial (NDI and COI) gene markers. Parasitology International 60(1):512. DOI 10.1016/j.parint.2010.09.003.

Arnqvist G., Mårtensson T. 1998. Measurement error in geometric morphometrics: empirical strategies to assess and reduce its impact on measure of shape. Acta Zoologica Academiae Scientiarum Hungaricae 44 (1-2):73-96.

Aroonroch R, Worawichawong S, Nitiyanant P, Kanchanapitak A, Bunyaratvej S. 2006. Hepatic fascioliasis due to Fasciola hepatica: a two-case report. Journal of the Medical Association of Thailand 89(10): 1770-1774.

Ashrafi K, Valero MA, Panova M, Periago MV, Massoud J, Mas-Coma S. 2006. Phenotypic analysis of adults of Fasciola hepatica, Fasciola gigantica and intermediate forms from the 
629

630

631

632

633

634

635

636

637

638

639

640

641

642

643

644

645

646

647

648

649

650

651

endemic region of Gilan, Iran. Parasitology International 55(4):249-260. DOI 10.1016/j.parint.2006.06.003.

Baylac M, Villemant C, Simbolotti G. 2003. Combining geometric morphometrics with pattern recognition for the investigation of species complexes. Biological Journal of the Linnean Society 80(1):89-98. DOI 10.1046/j.1095-8312.2003.00221.x.

Buranasin P, Harinasuta T. 1970. A case of fascioliasis in Thailand. The Southeast Asian Journal of Tropical Medicine and Public Health 1:146-147.

Burnaby TP. 1966. Growth invariant discriminant functions and generalized distances. Biometrics 22:96-110.

Chaichanasak P, Ichikawa M, Sobhon P, Itagaki T. 2012. Identification of Fasciola flukes in Thailand based on their spermatogenesis and nuclear ribosomal DNA, and their intraspecific relationships based on mitochondrial DNA. Parasitology International 61:545-549. DOI

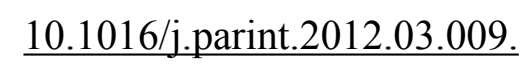

Darroch JN, Mosimann JE. 1985. Canonical and principal components of shape. Biometrika 72(2):241-252. DOI 10.1093/biomet/72.2.241.

Dobigny G, Baylac M, Denys C. 2002. Geometric morphometrics, neural networks and diagnosis of sibling Taterillus species (Rodentia, Gerbillinae). Biological Journal of the Linnean Society 77(3): 319-327. DOI 10.1046/j.1095-8312.2002.00074.x.

Dujardin JP, Kaba D, Solano P, Dupraz M, McCoy KD, Jaramillo-o N. 2014. Outline-based morphometrics, an overlooked method in arthropod studies?. Infection, Genetics and Evolution 28: 704-714. DOI 10.1016/j.meegid.2014.07.035

Dujardin JP, Dujardin S, Kaba D, Santillán-Guayasamín S, Villacís AG, Piyaselakul S, Sumruayphol S, Samung Y, Vargas RM. 2017. The maximum likelihood identification 
method applied to insect morphometric data. Zoological Systematics 42(1):46-58. DOI

653 10.11865/zs.201704.

654

655

656

657

658

659

660

661

662

663

664

665

666

667

668

669

670

671

672

Dujardin JP. 2019. A template-dependent semilandmarks treatment and its use in medical entomology. Infection, Genetics and Evolution 70:197-207. DOI 10.1016/j.meegid.2019. 03.002

Dujardin S, Dujardin JP. 2019. Geometric morphometrics in the cloud. Infection, Genetics and Evolution 70:189-196. DOI 10.1016/j.meegid.2019.02.018. Available at https://xyom.io.

Firmat C, Gomes-Rodrigues H, Renaud S, Claude J, Hutterer R, Garcia-Talavera F, Michaux J. 2010. Mandible morphology, dental microwear, and diet of the extinct giant rats Canariomys (Rodentia: Murinae) of the Canary Islands (Spain). Biological Journal of the Linnean Society 101(1):28-40. DOI 10.1111/j.1095-8312.2010.01488.x.

Good P. 2000. Permutation tests: a practical guide to resampling methods for testing hypotheses. New York, Springer

Halakou A, Khazan H, Bendehpour M, Kazemi B. 2017. Morphological study of Fasciola parasites isolated from cattle and sheep in Golestan Province (Iran). Novelty in Biomedicine 5(4):166-171.

Huang WY, He B, Wang CR, Zhu XQ. 2004. Characterisation of Fasciola species from Mainland China by ITS-2 ribosomal DNA sequence. Veterinary Parasitology 120(1-2): 7583. DOI 10.1016/j.vetpar.2003.12.006.

Ichikawa M, Itagaki T. 2010. Discrimination of the ITS1 types of Fasciola spp. based on a PCRRFLP method. Parasitology Research 106(3):757-761. DOI 10.1007/s00436-010-1724-2. 
673 Itagaki T, Ichinomiya M, Fukuda K, Fusyuku S, Carmona C. 2011. Hybridization experiments

674 indicate incomplete reproductive isolating mechanism between Fasciola hepatica and 675 Fasciola gigantica. Parasitology 138(10):1278-1284. DOI 10.1017/S0031182011000965.

676 Iyiola OA, Shittu O, Owolodun OA, Animasaun DA, Udeze AO. 2018. Morphometric 677 phenotypes and molecular identification of Fasciola species isolated from cattle in Ilorin, 678 North-Central Nigeria. Sri Lankan Journal of Biology 3(2):9-23. DOI 10.4038/sljb.v3i2.23. 679 Kanoksil W, Wattanatranon D, Wilasrusmee C, Mingphruedh S, Bunyaratvej S. 2006. 680 Endoscopic removal of one live biliary Fasciola gigantica. Journal of the Medical Association of Thailand 89(12):2150-2154.

682

683

684

685

686

Klingenberg CP, McIntyre GS. 1998. Geometric morphometrics of developmental instability: analyzing patterns of fluctuating asymmetry with Procrustes methods. Evolution 52: 13631375.

Klingenberg CP. 2016. Size, shape, and form: concepts of allometry in geometric morphometrics. Development genes and evolution 226(3):113-137. DOI 10.1007/s00427-016-0539-2.

Kuhl FP, Giardina CR. 1982. Elliptic Fourier features of a closed contour. Computer graphics and image processing 18(3):236-258. DOI 10.1016/0146-664X (82)90034-X.

Lin RQ, Dong SJ, Nie K, Wang CR, Song HQ, Li AX, Huang WY, Zhu XQ. 2007. Sequence analysis of the first internal transcribed spacer of rDNA supports the existence of the intermediate Fasciola between F. hepatica and F. gigantica in mainland China. Parasitology Research 101(3):813-817. DOI 10.1007/s00436-007-0512-0

Lorenz C, Ferraudo AS, Suesdek L. 2015. Artificial Neural Network applied as a methodology of mosquito species identification. Acta Tropica 152:165-169. DOI $\underline{10.1016 / j . a c t a t r o p i c a .2015 .09 .011 . ~}$ 
696

697

698

699

700

701

702

703

704

705

706

707

708

709

710

711

712

713

714

715

716

Lotfy WM, Brant SV, DeJong RJ, Le TH, Demiaszkiewicz A, Rajapakse RJ, Perera VB, Laursen JR, Loker ES. 2008. Evolutionary origins, diversification, and biogeography of liver flukes (Digenea, Fasciolidae). The American Journal of Tropical Medicine and Hygiene 79(2):248-255. DOI 10.4269/ajtmh.2008.79.248.

Mancuso S. 1999. Elliptic Fourier Analysis (EFA) and Artificial Neural Networks ( $A N N \mathrm{~s})$ for the identification of grapevine (Vitis vinifera L.) genotypes. Vitis 38(2):73-78.

Mas-Coma S, Bargues MD, Valero MA. 2005. Fascioliasis and other plant-borne trematode zoonoses. International Journal Parasitology 35(11-12):1255-1278. DOI 10.1016/j.jpara.2005.07.010

Mas-Coma S, Valero MA, Bargues MD. 2008. Effects of climate change on animal and zoonotic helminthiases. Revue Scientifique et Technique 27(2):443-457.

Mas-Coma S, Valero MA, Bargues MD. 2009a. Climate change effects on Trematodiases, with emphasis on zoonotic fascioliasis and schistosomiasis. Veterinary Parasitology 163(4):264280. DOI 10.1016/j.vetpar.2009.03.024.

Mas-Coma S, Valero MA, Bargues MD. 2009b. Fasciola, lymnaeids and human fascioliasis, with a global overview on disease transmission, epidemiology, evolutionary genetics, molecular epidemiology and control. Advances in Parasitology 69:41-146. DOI $\underline{10.1016 / \mathrm{S} 0065-308 \mathrm{X}(09) 69002-3 .}$.

Mas-Coma S, Bargues MD, Valero MA. 2018. Human fascioliasis infection sources, their diversity, incidence factors, analytical methods and prevention measures. Parasitology 145(13):1665-1699. DOI 10.1017/S0031182018000914

Peer] reviewing PDF | (2019:08:40125:2:0:NEW 7 Jan 2020) 
717 Mitteroecker P, Bookstein F. 2011. Linear Discrimination, Ordination, and the Visualization of 718 Selection Gradients in Modern Morphometrics. Evolutionary Biology 38(1):100-114. DOI

$719 \quad \underline{10.1007 / \mathrm{s} 11692-011-9109-8}$

720 Periago MV, Valero MA, Panova M, Mas-Coma S. 2006. Phenotypic comparison of allopatric 721 populations of Fasciola hepatica and Fasciola gigantica from European and African bovines using a computer image analysis system (CIAS). Parasitology Research 99(4):368-378. DOI

723 $10.1007 / \mathrm{s} 00436-006-0174-3$

724

725

726

727

728

729

730

731

732

733

734

735

736

737

738

739

Periago MV, Valero MA, Sayed ME, Ashrafi K, Wakeel AE, Mohamed MY, Desquesnes M, Curtale F, Mas-Coma S. 2008. First phenotypic description of Fasciola hepatica/ Fasciola gigantica intermediate forms from the human endemic area of the Nile Delta, Egypt. Infection, Genetics and Evolution 8(1):51-58. DOI 10.1016/j.meegid.2007.10.001

Polly PD, Head JJ. 2004. Maximum-likelihood identification of fossils: taxonomic identification of Quaternary marmots (Rodentia, Mammalia) and identification of vertebral position in the pipesnake Cylindrophis (Serpentes, Reptilia). In: Elewa AMT, editor. Morphometrics, Applications in Biology and Paleontology. Springer Science \& Business Media, 197-221.

Ripley BD. 1996. Pattern recognition and neural networks. Cambridge: Cambridge University Press.

Rohlf FJ, Marcus LF. 1993. A revolution in morphometrics. Trends in Ecology \& Evolution 8(4):129-132. DOI 10.1016/0169-5347(93)90024-J.

Rohlf FJ, Slice D. 1990. Extensions of the procrustes method for the optimal superimposition of landmarks. Systematics Zoology 39(1):40-59. DOI 10.2307/2992207.

Rosenblatt F. 1958. The Perceptron: A theory of statistical separability in cognitive systems. Cornell Aeronautical Laboratory, Report No. VG1196-G-1. 
740 Rumelhart DE, Hinton G E, Williams RJ. 1988. Learning representations by back-propagating

741

742

743

744

745

746

747

748

749

750

751

752

753

754

755

756

757

758

759

760

761

762 errors. Nature 323:533-536.

Siribat P, Dekumyoy P, Komalamisra C, Sumruayphol S, Thaenkham U. 2018. Molecular identification of Fasiola spp. representative samples from Thailand based on PCR-RFLP. The Journal of Tropical Medicine and Parasitology 41:1-7.

Shafiei R, Sarkari B, Sadjjadi SM, Mowlavi GR, Moshfe A. 2014. Molecular and morphological characterization of Fasciola spp. isolated from different host species in a newly emerging focus of human fascioliasis in Iran. Veterinary Medicine International 405740. DOI 10.1155/2014/405740

Sheets HD, Covino KM, Panasiewicz JM, Morris SR. 2006. Comparison of geometric morphometric outline methods in the discrimination of age-related differences in feather shape. Frontiers in Zoology 3(1):2-15. DOI 10.1186/1742-9994-3-15.

Soda KJ, Slice DE, Naylor GJP. 2017. Artificial neural networks and geometric morphometric methods as a means for classification: A case-study using teeth from Carcharhinus sp. (Carcharhinidae). Journal of Morphology 278(1):131-141. DOI 10.1002/jmor.20626).

Sonnenschein A, VanderZee D, Pitchers WR, Chari S, Dworkin I. 2015. An image database of Drosophila melanogaster wings for phenomic and biometric analysis. Gigascience 4:25. DOI 10.1186/s13742-015-0065-6.

Srihakim S, Pholpark M. 1991. Problem of fascioliasis in animal husbandry in Thailand. The Southeast Asian Journal of Tropical Medicine and Public Health 22(Suppl):352-355.

Srimuzipo P, Komalamisra C, Choochote W, Jitpakdi A, Vanichthanakorn, P, Keha P, Riyong D, Sukontason K, Komalamisra N, Sukontason K. 2000. Comparative morphometry, morphology of egg and adult surface topography under light and scanning 
763

764

765

766

767

768

769

770

771

772

773

774

775

776

777

778

779

780

781

782

783

784

785

electron microscopics, and metaphase karyotype among three size-races of Fasciola gigantica in Thailand. The Southeast Asian Journal of Tropical Medicine and Public Health 31(2):366-373.

Tippawangkosol P, Saingamsook J, Kijdamrongthum P, Thinrungroj N, Jariyawat K, Somboon P. 2017. Endoscopic management of biliary fascioliasis and identification of Fasciola fluke based on mitochondrial DNA: A case report from Thailand. The Southeast Asian Journal of Tropical Medicine and Public Health 48(3):524-531.

Valero MA, Marcos MD, Mas-Coma S. 1996. Mathematical model for the ontogeny of Fasciola hepatica in the definitive host. Research \& Reviews in Parasitology 56:13-20.

Valero MA, Panova M, Mas-Coma S. 2005. Phenotypic analysis of adults and eggs of Fasciola hepatica by computer image analysis system. Journal of Helminthology 79(3):217-225. DOI $\underline{10.1079 / \mathrm{JOH} 2005301 .}$.

Van Bocxlaer B, Schultheiß R. 2010. Comparison of morphometric techniques for shapes with few homologous landmarks based on machine-learning approaches to biological discrimination. Paleobiology 36(3):497-515. DOI 10.1666/08068.1.

Varea Sánchez M, Bastir M, Roldan ER. 2013. Geometric morphometrics of rodent sperm head shape. PloS One 8(11): e80607. DOI 10.1371/journal.pone.0080607.

Waikagul J, Thaenkham U. 2014. Approaches to research on the systematics of fish-borne trematodes. Academic Press.

Wannasan A, Khositharattanakool P, Chaiwong P, Piangjai S, Uparanukraw P, Morakote N. 2014. Identification of Fasciola species based on mitochondrial and nuclear DNA reveals the co-existence of intermediate Fasciola and Fasciola gigantica in Thailand. Experimental Parasitology 146: 64-70. DOI 10.1016/j.exppara.2014.09.006. 
786 Yang HP, Ma CS, Wen H, Zhan QB, Wang XL. 2015. A tool for developing an automatic insect

787 identification system based on wing outlines. Scientific report 5:12786. DOI $788 \quad 10.1038 / \operatorname{srep} 12786$. 


\section{Figure 1}

Geometric morphometric analysis of Fasciola adult worms

(A) Contour digitized on Fasciola spp. body for outline-based geometric morphometrics analysis, (B) Position of 5 landmarks digitized on Fasciola spp. body for landmark-based geometric morphometric analysis. 


\section{A}

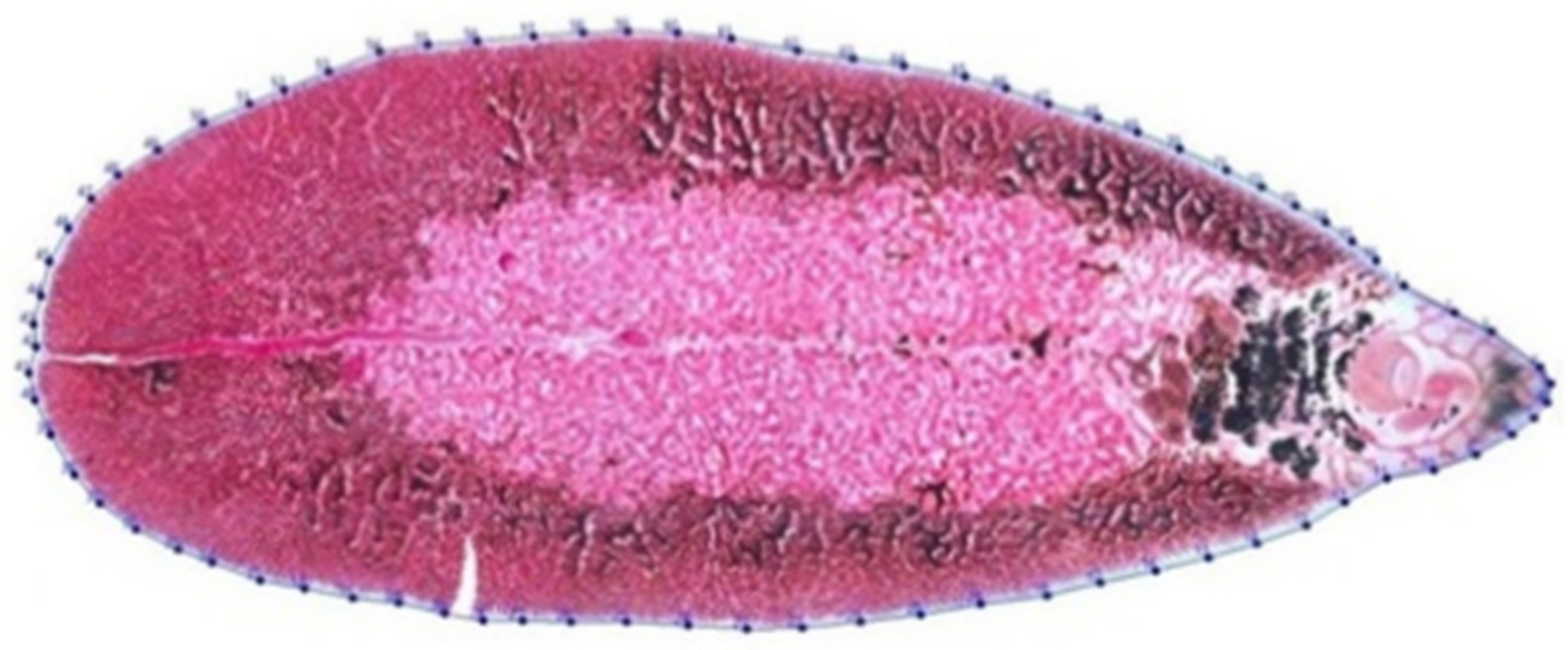

$1 \mathrm{~cm}$

\section{B}

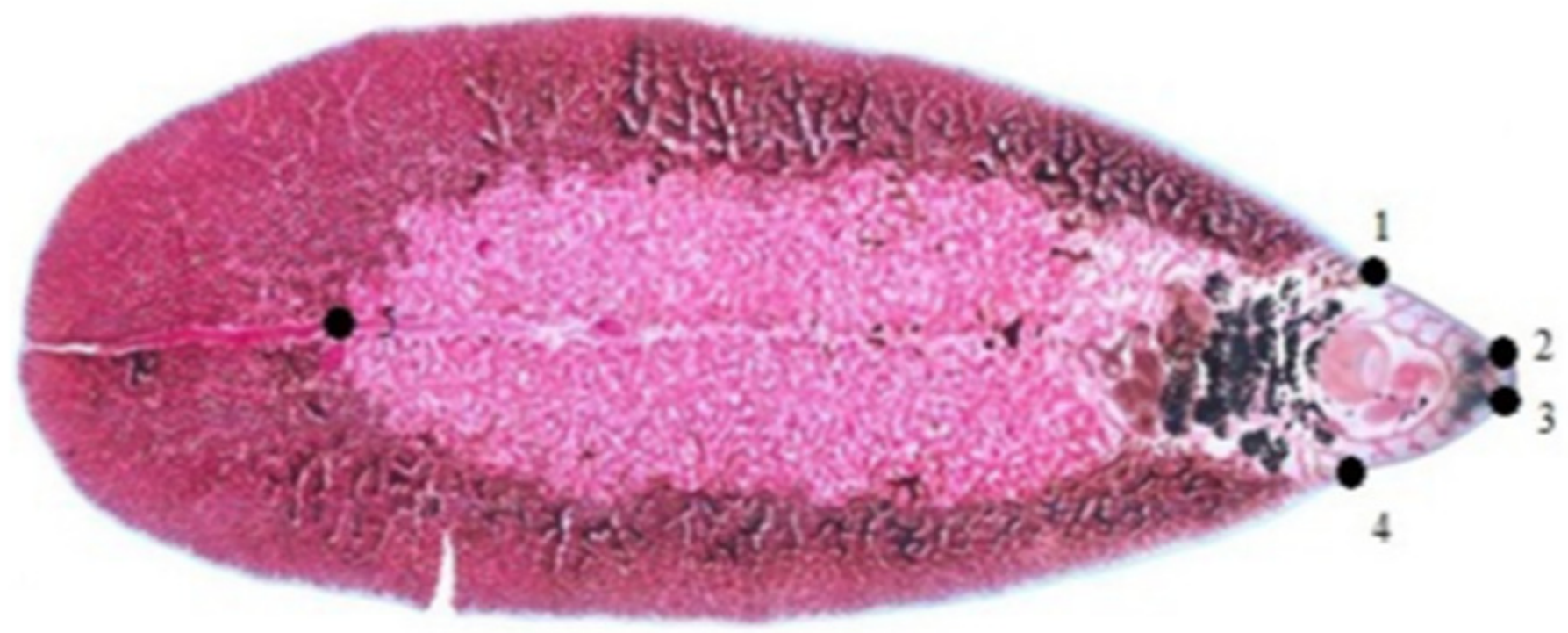

$1 \mathrm{~cm}$ 


\section{Figure 2}

Global size of Fasciola adult worms

(A) Quantile boxes showing body perimeter variation obtained after outline-based geometric morphometric analysis. Each box shows the group median that separates the 25th and 75th quartiles. Dots on the left represent individuals. (B) Variation of body centroid size of Fasciola spp. from landmark-based GM analysis. Each box shows the group median that separates the 25th and 75th quartiles. Dots on the left represent individuals. 
A

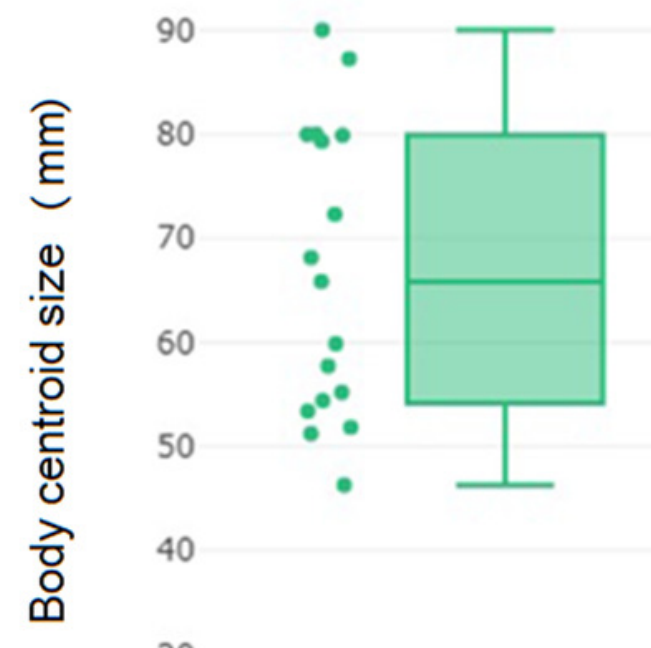

F. gigantica
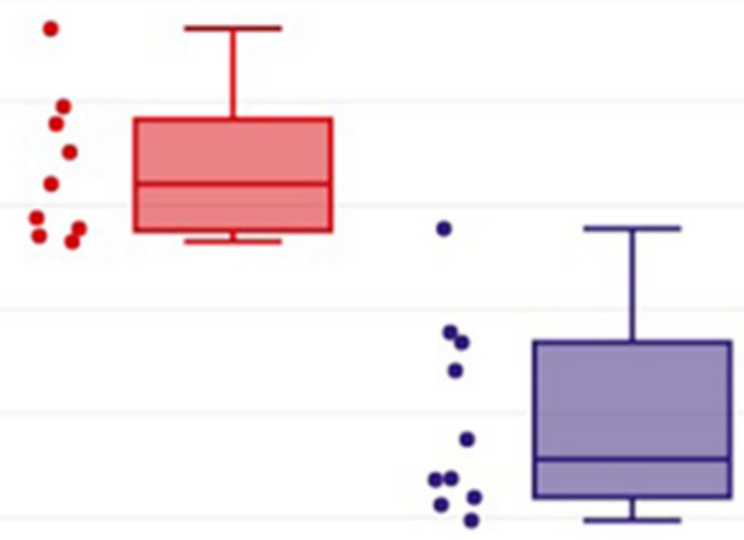

F. hepatica

B

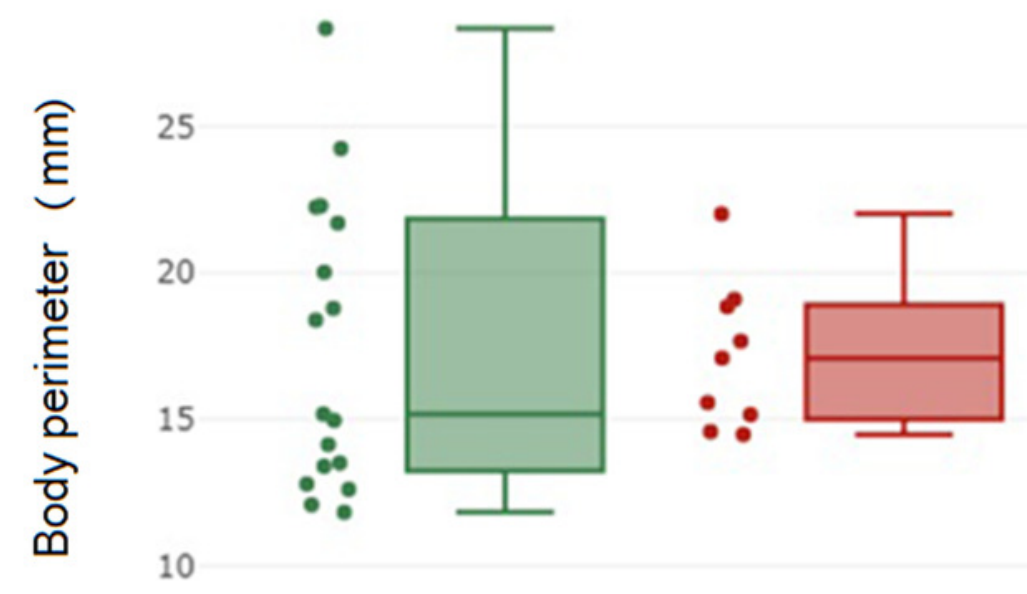

F. gigantica
F. hepatica

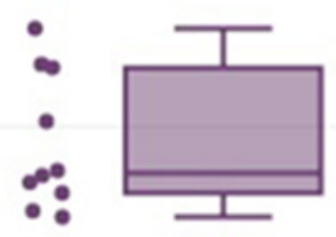

Fasciola intermediate form 


\section{Figure 3}

Shape variation of Fasciola spp. based on the outline-based analysis

$<!--[i f$ !supportLists]-->(A) <!--[endif]--> Mean shape of whole body contour of $F$. gigantica, $F$. hepatica, and Fasciola intermediate forms. (B) Outline-based discriminant analysis. Factor map of the two discriminant factor (DFs) derived from shape variables for Fasciola species. Each point represents an individual. The horizontal axis is the first DF; the vertical axis is the second DF; their cumulated contributions reach $100 \%$ of the total variation. 


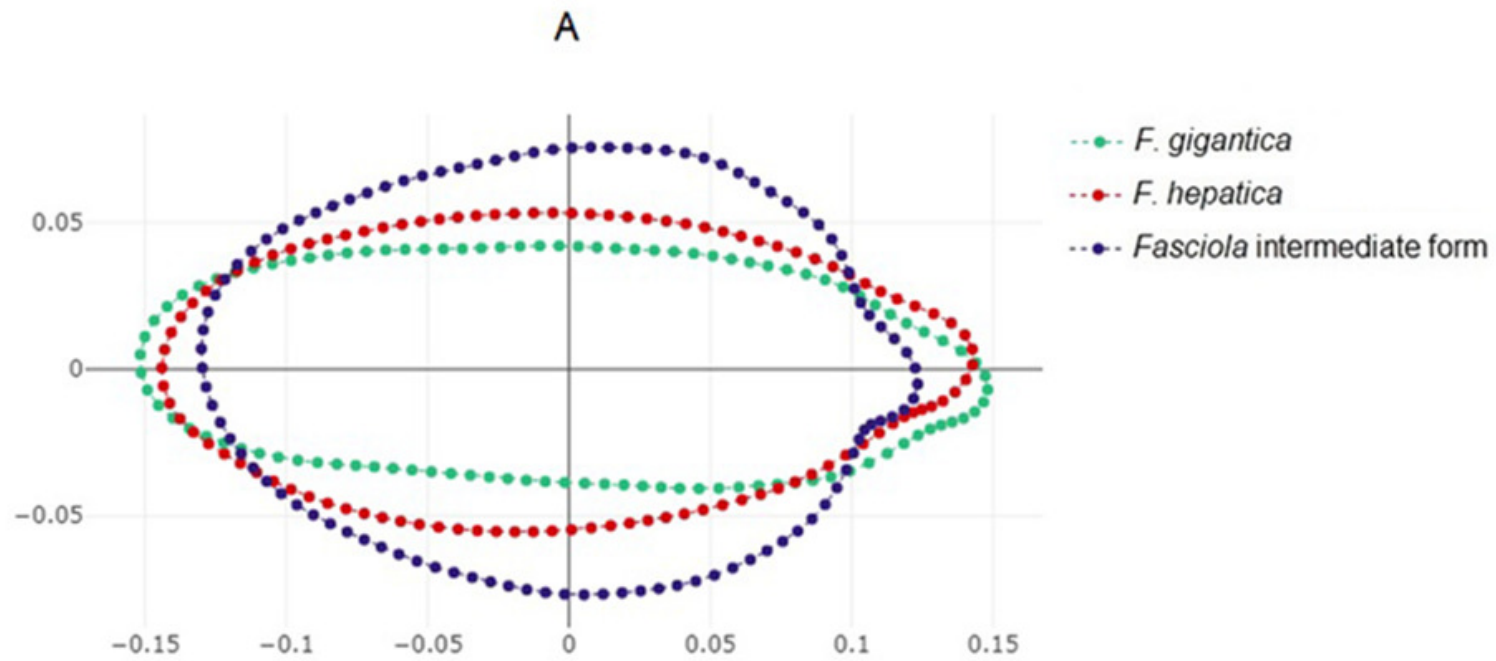

B

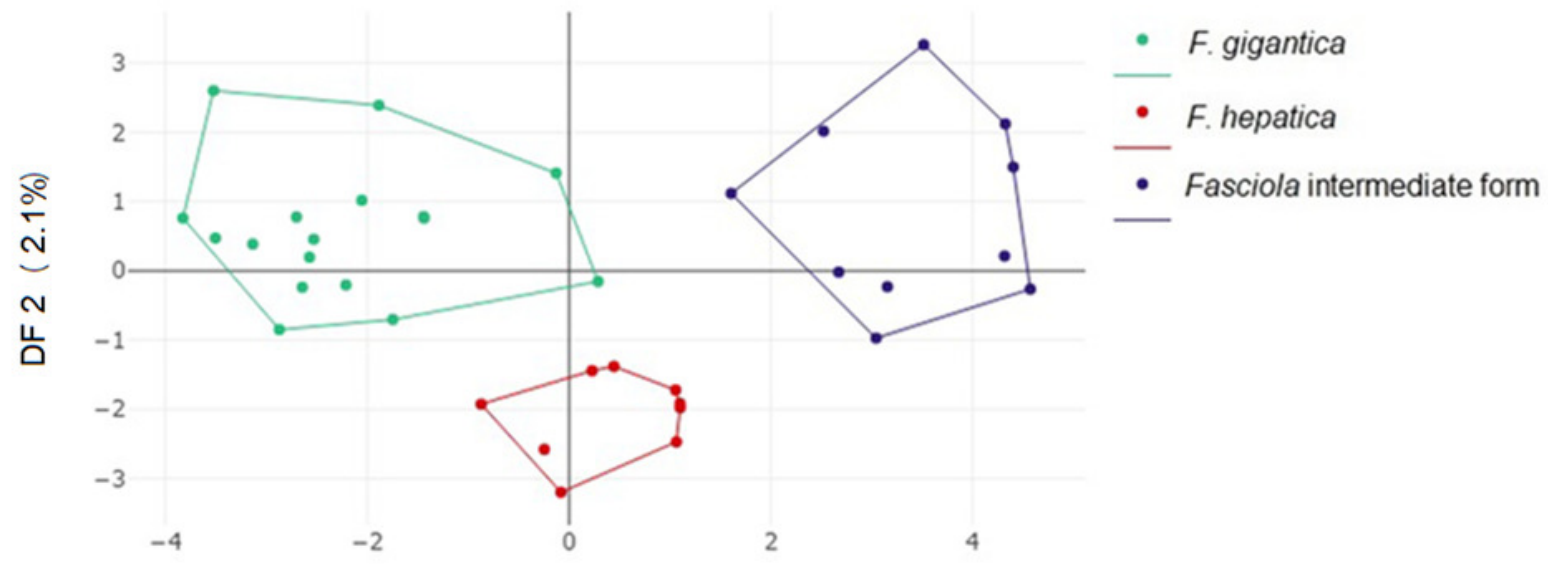

DF $1(97.9 \%)$ 


\section{Figure 4}

Shape variation of Fasciola spp. based on landmark-based analyses

(A) Average positions of anatomical landmarks (dots) after Procrustes superposition, representing the mean shapes of $F$. gigantica, F. hepatica, and of Fasciola intermediate forms, (B) Landmark-based discriminant analysis. Factor map of the two discriminant factors (DFs) derived from final shape variables of F. gigantica, F. hepatica, and Fasciola intermediate forms. Each point represents an individual. The horizontal axis is the first DF; the vertical axis is the second DF, their cumulated contributions reach $100 \%$ of the total variation. 
A

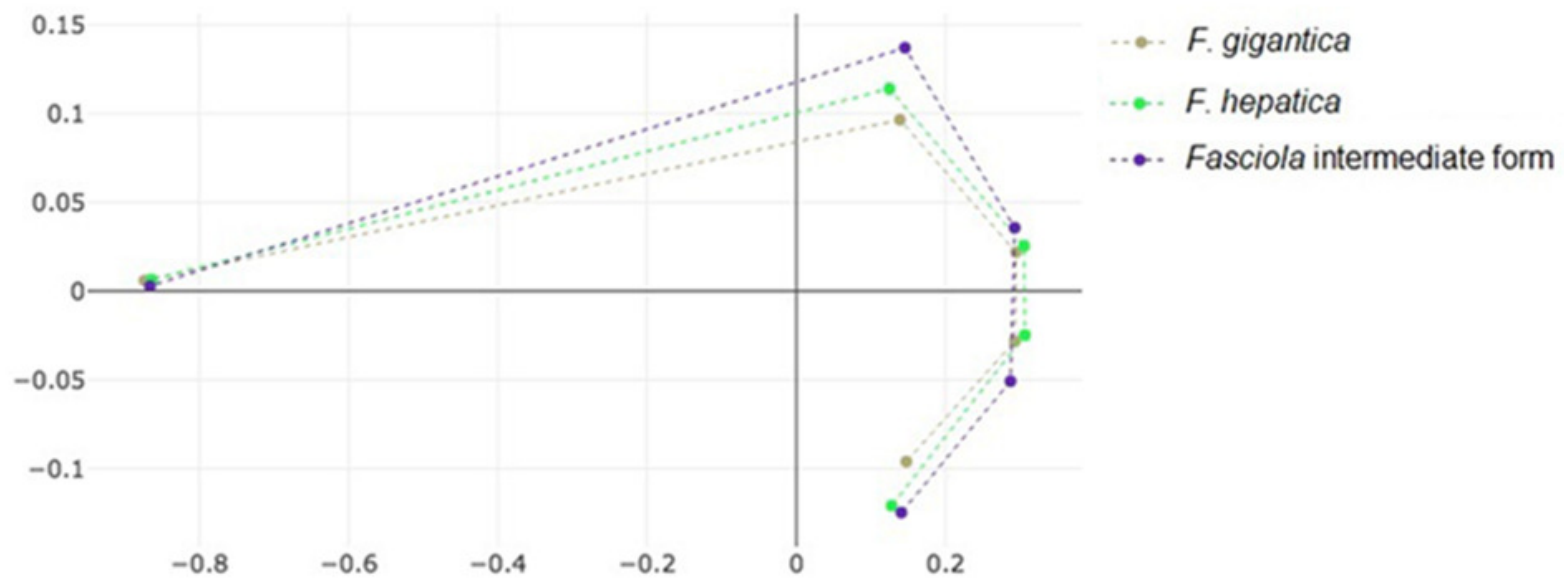

B

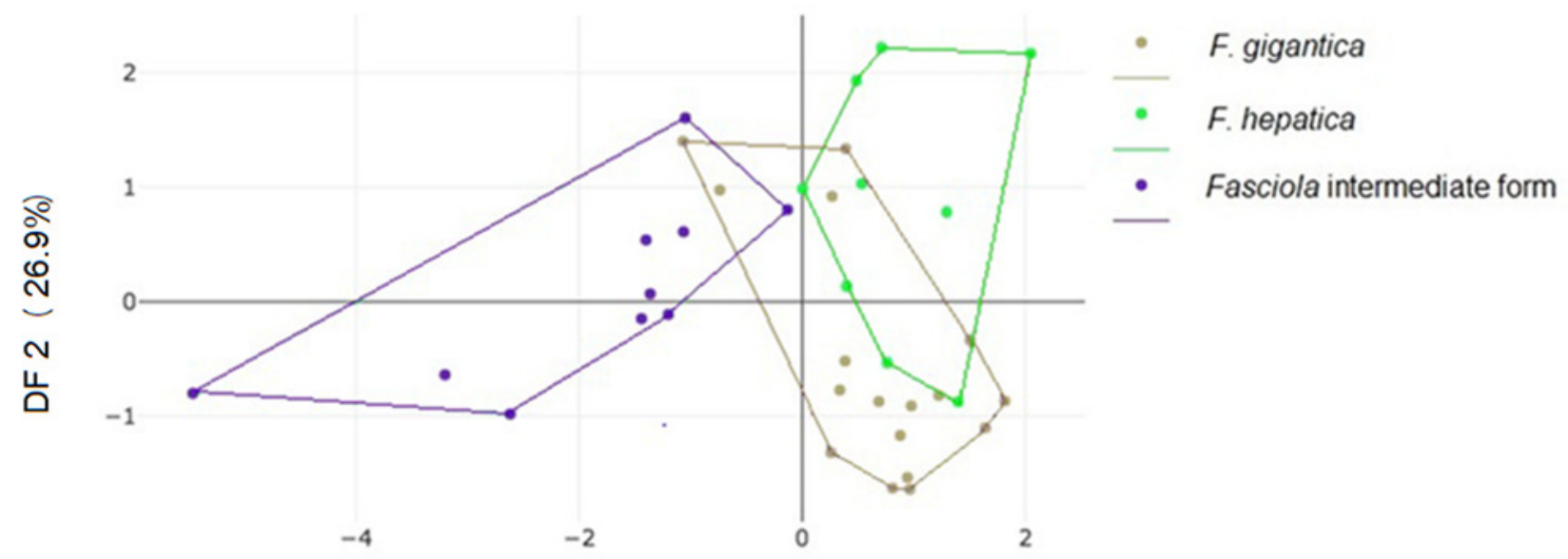

DF $1(73.1 \%)$ 


\section{Table $\mathbf{1}$ (on next page)}

Visual identification versus molecular typing of field specimen

The visual examination of external morphology could not recognize any of the 16

intermediate forms, most of them (14) wrongly identified as $F$. hepatica. A minor part of $F$. gigantica (13) was misidentified also with $F$. hepatica. 
1 Table 1. Visual identification versus molecular typing of field specimen

2

\begin{tabular}{ccc}
\hline Species & External Morphology & PCR-RFLP \\
\cline { 2 - 3 } F. gigantica & 63 & 74 \\
Fasciola intermediate forms & 0 & 16 \\
F. hepatica & 27 & 0 \\
\hline
\end{tabular}

3

4

5 The visual examination of external morphology could not recognize any of the 16 intermediate

6 forms, most of them (14) wrongly identified as $F$. hepatica. A minor part of $F$. gigantica (13)

7 was misidentified also with $F$. hepatica. 


\section{Table 2 (on next page)}

Traditional morphometric analyses of Fasciola species

Fg, Fasciola gigantica; Fh, Fasciola hepatica; Fif, Fasciola intermediate forms. M, mean. n, sample size; Min, minimum, Max, maximum; SD, standard deviation; BL, Body length, $B L ;$ BW, Body width, BW; BWOv, BW at ovary level; BP, Body perimeter; CL, Cone length; CW, Cone width; Osmax, Oral sucker maximum diameter; Osmin, Oral sucker minimum diameter; Vsmax, Ventral sucker maximum diameter; Vsmin, Ventral sucker minimum diameter; A-VS, Distance between anterior end of body and VS; OS-VS, Distance between suckers; VS-Vit, Distance between VS and union of vitelline glands; Vit-P, Distance between Vit and posterior end of body; VS-P, Distance between VS and posterior end of body; PhL, Pharynx length; PhW, Pharynx width; TL, Testicular space length; TW, Testicular space width; TP, Testicular space perimeter. 
1 Table 2. Traditional morphometric analyses of Fasciola species

2

\begin{tabular}{ccccc}
\hline & $F g$ & $F i f$ & $F h$ & $P$ \\
Character & $\mathrm{n}=17$ & $\mathrm{n}=10$ & $\mathrm{n}=9$ & \\
\hline
\end{tabular}

$\begin{array}{lllllllllllll}1 . \mathrm{BL} & 29.9 & 20.8-42.1 & 6.7 & 16.0 & 10.9-23.2 & 3.6 & 28.2 & 23.8-33.3 & 3.1 & 0.000 & 0.787 & 0.000\end{array}$

$\begin{array}{lllllllllllll}\text { 2. } \mathrm{BW} & 7.4 & 4.8-10.9 & 1.9 & 8.6 & 5.9-12.8 & 2.2 & 10.2 & 8.2-12.9 & 1.5 & 0.141 & 0.001 & 0.107\end{array}$

$\begin{array}{lllllllllllll}\text { 3. BWOv } & 4.4 & 3.3-5.9 & 0.7 & 5.1 & 3.3-7.7 & 1.3 & 5.1 & 4.2-5.6 & 0.5 & 0.113 & 0.063 & 0.746\end{array}$

$\begin{array}{lllllllllllll}\text { 4. } \mathrm{BP} & 65.4 & 46.3-88.2 & 13.6 & 37.5 & 29.2-57.5 & 9.0 & 63.3 & 54.6-74.8 & 6.6 & 0.000 & 0.900 & 0.000\end{array}$

$\begin{array}{lllllllllllll}\text { 5. CL } & 2.9 & 2.4-3.7 & 0.4 & 1.6 & 1.2-2.1 & 0.3 & 3.4 & 2.6-3.9 & 0.4 & 0.000 & 0.241 & 0.000\end{array}$

$\begin{array}{lllllllllllll}\text { 6. } \mathrm{CW} & 3.3 & 2.4-4.1 & 0.5 & 2.4 & 1.80-3.35 & 0.5 & 4.1 & 3.4-4.6 & 0.4 & 0.003 & 0.021 & 0.000\end{array}$

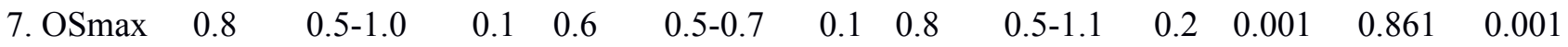

$\begin{array}{lllllllllllll}\text { 8. OSmin } & 0.5 & 0.3-0.7 & 0.1 & 0.4 & 0.3-0.6 & 0.1 & 0.6 & 0.3-0.8 & 0.2 & 0.399 & 0.089 & 0.023\end{array}$

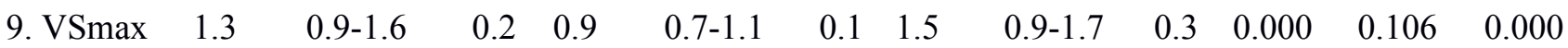

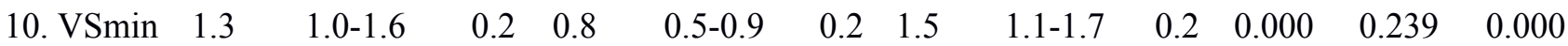

$\begin{array}{lllllllllllll}\text { 11. A-VS } & 2.2 & 1.5-2.9 & 0.1 & 1.5 & 1.1-2.3 & 0.3 & 2.9 & 2.6-3.5 & 0.3 & 0.000 & 0.032 & 0.000\end{array}$

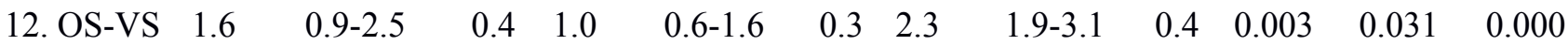

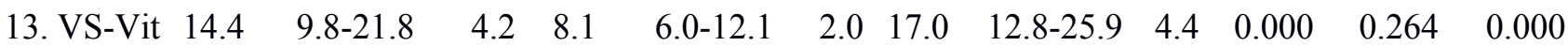

$\begin{array}{lllllllllllll}\text { 14. Vit-P } & 13.5 & 5.8-33.4 & 7.1 & 4.1 & 1.3-8.1 & 1.7 & 7.7 & 5.8-10.8 & 1.4 & 0.000 & 0.059 & 0.014\end{array}$

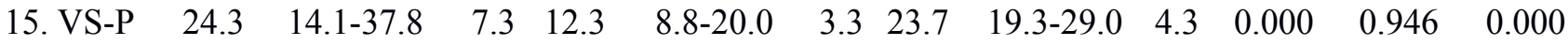

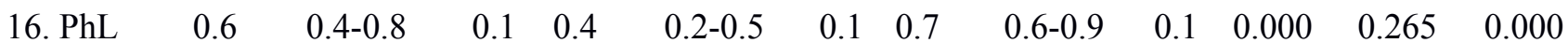

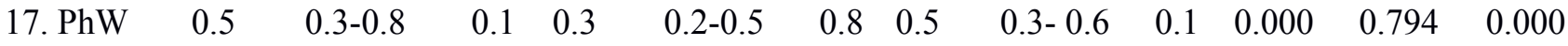

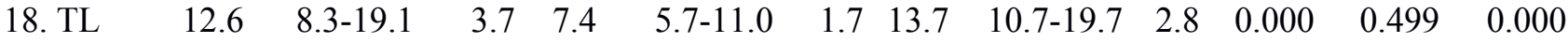




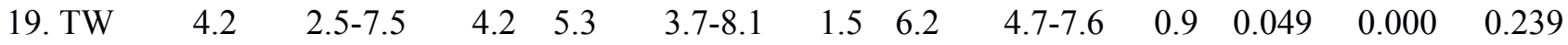

$\begin{array}{lllllllllllll}20 . \mathrm{TP} & 31.6 & 22.2-43.1 & 7.6 & 25.0 & 17.8-33.2 & 6.1 & 35.9 & 30.6-46.1 & 4.7 & 0.019 & 0.163 & 0.000\end{array}$

3

4 Fg, Fasciola gigantica; Fh, Fasciola hepatica; Fif, Fasciola intermediate forms. M, mean. n, 5 sample size; Min, minimum, Max, maximum; SD, standard deviation; BL, Body length, BL; BW, 6 Body width, BW; BWOv, BW at ovary level; BP, Body perimeter; CL, Cone length; CW, Cone 7 width; Osmax, Oral sucker maximum diameter; Osmin, Oral sucker minimum diameter; Vsmax, 8 Ventral sucker maximum diameter; Vsmin, Ventral sucker minimum diameter; A-VS, Distance 9 between anterior end of body and VS; OS-VS, Distance between suckers; VS-Vit, Distance 10 between VS and union of vitelline glands; Vit-P, Distance between Vit and posterior end of body; 11 VS-P, Distance between VS and posterior end of body; PhL, Pharynx length; PhW, Pharynx width; 12 TL, Testicular space length; TW, Testicular space width; TP, Testicular space perimeter. 


\section{Table 3 (on next page)}

Validated classifications according to three methods and three morphometric approaches (Traditional, Outlines and Landmarks).

Percentages of correct classification according to data sets and classification methods. Fg, Fasciola gigantica; Fg, Fasciola hepatica; Fif, Fasciola intermediate forms; LN, natural logarithms; LSR, Log-Shape-Ratios; OD, outline data, here Normalized Elliptic Fourier coefficients; LD, Procrustes residuals (orthogonal projections). ANN, artificial neural network. For ANN, the results are presented as averages over 30 sessions and, between square brackets, the standard deviation; $M x L$, maximum likelihood validated reclassification: between brackets, the number of PC giving the best reclassification score; the Dist (PC) the Mahalanobis distance-based validated method used the 8 first principal components of the variables $L N$ and $O D$, and the 6 PC of ORP. 
1 Table 3. Validated classifications according to three methods and three morphometric approaches 2 (Traditional, Outlines and Landmarks).

\begin{tabular}{|c|c|c|c|c|c|c|c|}
\hline \multirow[t]{2}{*}{ Methods } & \multirow{2}{*}{$\begin{array}{l}\text { Samples/ } \\
\text { Variables }\end{array}$} & \multicolumn{4}{|c|}{ Traditional } & \multirow{3}{*}{$\begin{array}{c}\text { Outlines } \\
30 \text { OD }\end{array}$} & \multirow{3}{*}{$\begin{array}{c}\text { Landmarks } \\
6 \text { LD }\end{array}$} \\
\hline & & $20 \mathrm{LN}$ & LSR & $12 \mathrm{LN}$ & LSR & & \\
\hline & & & $(20 \mathrm{LN})$ & & (12 LN) & & \\
\hline \multirow[t]{4}{*}{ ANN } & $17 F g$ & $13.63[0.85]$ & 13.5 [1.01] & $13.47[1.04]$ & 14.17 [1.05] & $14.96[0.69]$ & $10.81[2]$ \\
\hline & $9 F h$ & $8.4[0.67]$ & $7.6[0.72]$ & $8.43[0.68]$ & $7.73[0.69]$ & $7.13[0.85]$ & 5.65 [1.21] \\
\hline & 10 Fif & $9.97[0.18]$ & $9.93[0.25]$ & $9.83[0.46]$ & $8.43[0.73]$ & $9.96[0.20]$ & 8.33 [0.95] \\
\hline & 36 & $89 \%[2.8]$ & $86 \%[3.5]$ & $88 \%$ [3.5] & $84 \%[2.8]$ & $89 \%[3.5]$ & $68 \%[5.6]$ \\
\hline \multirow[t]{4}{*}{$M x L(P C)$} & $17 F g$ & 16 & 15 & 17 & 15 & 14 & 9 \\
\hline & $9 F h$ & 7 & 7 & 7 & 7 & 8 & 6 \\
\hline & 10 Fif & 9 & 10 & 8 & 9 & 10 & 6 \\
\hline & 36 & $89 \%(3)$ & $89 \%(4)$ & $89 \%(4)$ & $86 \%$ & $89 \%(4)$ & $58 \%(3)$ \\
\hline \multirow[t]{4}{*}{$\operatorname{Dist}(P C)$} & $17 F g$ & 15 & 15 & 17 & 17 & 16 & 13 \\
\hline & $9 F h$ & 8 & 8 & 8 & 9 & 8 & 5 \\
\hline & 10 Fif & 10 & 10 & 10 & 9 & 10 & 7 \\
\hline & 36 & $92 \%$ & $92 \%$ & $97 \%$ & $97 \%$ & $94 \%$ & $69 \%$ \\
\hline
\end{tabular}

3

4 Percentages of correct classification according to data sets and classification methods. $F g$,

5 Fasciola gigantica; Fg, Fasciola hepatica; Fif, Fasciola intermediate forms; LN, natural

6 logarithms; LSR, Log-Shape-Ratios; OD, outline data, here Normalized Elliptic Fourier

7 coefficients; LD, Procrustes residuals (orthogonal projections). $A N N$, artificial neural network. For 
8 ANN, the results are presented as averages over 30 sessions and, between square brackets, the 9 standard deviation; $M x L$, maximum likelihood validated reclassification: between brackets, the 10 number of PC giving the best reclassification score; the Dist (PC) the Mahalanobis distance-based 11 validated method used the 8 first principal components of the variables LN and OD, and the 6 PC 12 of ORP. 


\section{Table 4(on next page)}

Global sizes of Fasciola spp.

Fg, Fasciola gigantica; Fif, Fasciola intermediate forms; Fh, Fasciola hepatica; n, number of specimen; Min, minimum; Max, maximum; SD Standard deviation. Perimeter and square-root area as computed by the outline-based technique, and centroid size by the Generalized Procrustes Analysis (landmark-based technique). 
1 Table 4. Global sizes of Fasciola spp.

2 Table 4a. Body perimeter and Square-root area of Fasciola spp.

\begin{tabular}{|c|c|c|c|c|c|}
\hline Species & $\mathrm{n}$ & Mean & Min & Max & SD \\
\hline & \multicolumn{5}{|c|}{ Body Perimeter } \\
\hline$F g$ & 17 & 65.44 & 45.22 & 88.47 & 13.96 \\
\hline$F h$ & 9 & 62.25 & 55.55 & 75.40 & 6.88 \\
\hline \multirow[t]{2}{*}{ Fif } & 10 & 38.67 & 29.05 & 56.83 & 9.14 \\
\hline & \multicolumn{5}{|c|}{ Square-root area } \\
\hline$F g$ & 17 & 13.5 & 9.04 & 17.89 & 2.54 \\
\hline$F h$ & 9 & 14.3 & 10.64 & 14.68 & 1.76 \\
\hline Fif & 10 & 9.8 & 5.20 & 10.40 & 2.33 \\
\hline
\end{tabular}

3

4

5 Table 4b. Centroid size of Fasciola spp.

\begin{tabular}{cccccc}
\hline Species & $\mathrm{n}$ & Mean & Min & Max & SD \\
\cline { 2 - 5 }$F g$ & 17 & 17.44 & 11.83 & 28.33 & 4.98 \\
Fh & 9 & 17.16 & 14.48 & 22.00 & 2.52 \\
Fif & 10 & 9.45 & 6.93 & 13.36 & 2.31 \\
\hline
\end{tabular}

6

7 Fg, Fasciola gigantica; Fif, Fasciola intermediate forms; Fh, Fasciola hepatica; n, number of

8 specimen; Min, minimum; Max, maximum; SD Standard deviation. Perimeter and square-root

9 area as computed by the outline-based technique, and centroid size by the Generalized Procrustes

10 Analysis (landmark-based technique). 


\section{Table 5(on next page)}

Non-parametric comparisons of global size estimations, 1000 permutations ( $P$-values)

$P$, percentage of values equal or higher than the observed differences of mean values between species after 1000 random permutations. It is the empirical risk of error saying that the differences are significant. BP, body perimeter. 
1 Table 5. Non-parametric comparisons of global size estimations, 1000 permutations ( $P$-values)

\begin{tabular}{lll}
\hline \multicolumn{1}{c}{ Species } & Centroid size & BP / Square-root area \\
\cline { 2 - 3 } F. gigantica / F. hepatica & $P=0.909$ & $P=0.650 / P=0.555$ \\
F. gigantica / F. intermediate forms & $P<0.001$ & $P<0.001 / P=0.003$ \\
F. intermediate forms / F. hepatica & $P<0.001$ & $P<0.004 / P<0.001$
\end{tabular}

2

$3 P$, percentage of values equal or higher than the observed differences of mean values between

4 species after 1000 random permutations. It is the empirical risk of error saying that the

5 differences are significant. BP, body perimeter. 


\section{Table 6(on next page)}

Mahalanobis distance and $P$-value for outline-based and landmark-based GM analyses

Fg, Fasciola gigantica; Fg, Fasciola hepatica; Fif, Fasciola intermediate forms 
1 Table 6. Mahalanobis distance and $P$-value for outline-based and landmark-based GM analyses

\begin{tabular}{|c|c|c|c|c|c|c|}
\hline \multirow[t]{2}{*}{ Species } & \multicolumn{3}{|c|}{ Outline-based GM } & \multicolumn{3}{|c|}{ Landmark-based GM } \\
\hline & $F g$ & $F h$ & Fif & $F g$ & $F h$ & Fif \\
\hline$F g$ & 0.00 & & & 0.00 & & \\
\hline$F h$ & $\begin{array}{c}2.28 \\
(p=0.108)\end{array}$ & 0.00 & & $\begin{array}{c}1.68 \\
(p=0.063)\end{array}$ & 0.00 & \\
\hline Fif & $\begin{array}{c}4.42 \\
(p<0.001)\end{array}$ & $\begin{array}{c}4.23 \\
(p=0.001)\end{array}$ & 0.00 & $\begin{array}{c}2.85 \\
(p<0.001)\end{array}$ & $\begin{array}{c}2.86 \\
(p<0.001)\end{array}$ & 0.00 \\
\hline
\end{tabular}

2

3 Fg, Fasciola gigantica; Fg, Fasciola hepatica; Fif, Fasciola intermediate forms

4

5 


\section{Table 7 (on next page)}

Scores of validated classification using as input for the multilayer perceptron either logtransformed linear measurements (LN) alone, or a combination of LN with outline data.

Percentages are the average of 30 ANN classification sessions (see Supplementary data 1), with the standard deviation between square brackets. LN, log-transformed linear measurements; OD, outline data, i.e., normalized elliptic Fourier coefficients. The first row may be read as follows: with only four log-transformed linear measurements (4LN, first column), $78 \%$ of correct species assignment was obtained after validated classification (second column), 88\% when these data were combined with outline data (third column). 
1 Table 7. Scores of validated classification using as input for the multilayer perceptron either log-

2 transformed linear measurements (LN) alone, or a combination of LN with outline data.

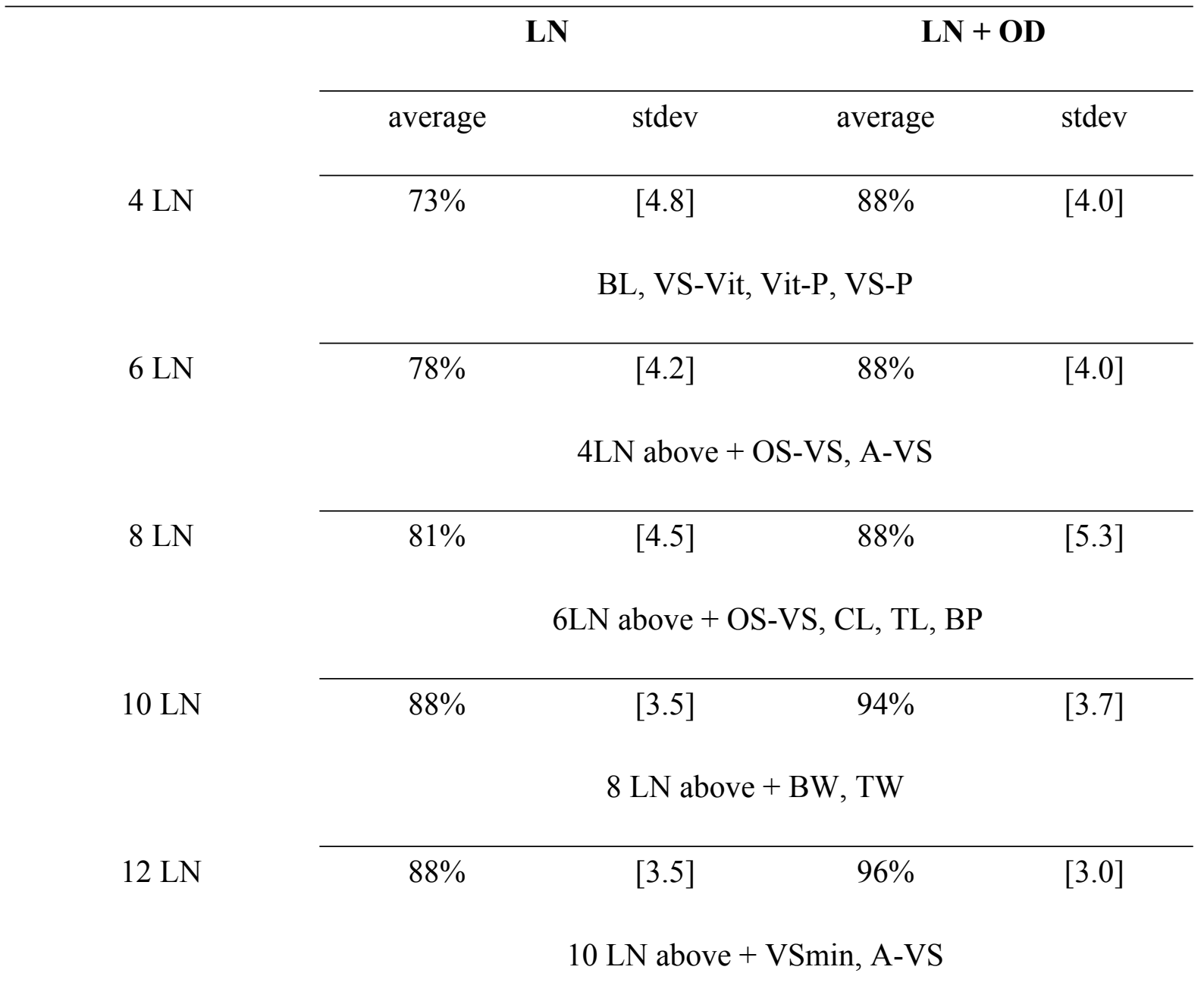

3

4 Percentages are the average of 30 ANN classification sessions (see Supplementary data 1), with

5 the standard deviation between square brackets. LN, log-transformed linear measurements; OD, 6 outline data, i.e., normalized elliptic Fourier coefficients. The first row may be read as follows:

7 with only four log-transformed linear measurements (4LN, first column), $78 \%$ of correct species 8 assignment was obtained after validated classification (second column), $88 \%$ when these data were 9 combined with outline data (third column). 